\title{
Taposiris Magna et Plinthine (2020)
}

Deux villes de Maréotide

Bérangère Redon, Marie-Françoise Boussac, Essam Ahmed, Charlène Bouchaud, Maël Crépy, Sylvain Dhennin, Mennat-Allah El Dorry, Paul François, Louis Dautais, Joachim Le Bomin, Louis Manière, Julie Marchand, Séverine Marchi, Clémence Pagnoux, Mikaël Pesenti, Alexandre Rabot, Gonca Şenol, Claire Somaglino, Matthieu Vanpeene et Noémi Villars

\section{(2) OpenEdition}

Édition électronique

URL : https://journals.openedition.org/baefe/2825

DOI : $10.4000 /$ baefe. 2825

ISSN : 2732-687X

Éditeur

ResEFE

Référence électronique

Bérangère Redon, Marie-Françoise Boussac, Essam Ahmed, Charlène Bouchaud, Maël Crépy, Sylvain Dhennin, Mennat-Allah El Dorry, Paul François, Louis Dautais, Joachim Le Bomin, Louis Manière, Julie Marchand, Séverine Marchi, Clémence Pagnoux, Mikaël Pesenti, Alexandre Rabot, Gonca Şenol, Claire Somaglino, Matthieu Vanpeene et Noémi Villars, « Taposiris Magna et Plinthine (2020) » [notice archéologique], Bulletin archéologique des Écoles françaises à l'étranger [En ligne], Égypte, mis en ligne le 30 mai 2021, consulté le 27 juillet 2021. URL : http://journals.openedition.org/baefe/2825 ; DOI https://doi.org/10.4000/baefe.2825

Ce document a été généré automatiquement le 27 juillet 2021

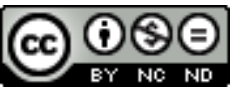

Le Bulletin archéologique des Écoles françaises à l'étranger est mise à disposition selon les termes de la Licence Creative Commons Attribution - Pas d'Utilisation Commerciale - Pas de Modification 4.0 International. 


\title{
Taposiris Magna et Plinthine (2020)
}

Deux villes de Maréotide

\author{
Bérangère Redon, Marie-Françoise Boussac, Essam Ahmed, Charlène \\ Bouchaud, Maël Crépy, Sylvain Dhennin, Mennat-Allah El Dorry, Paul \\ François, Louis Dautais, Joachim Le Bomin, Louis Manière, Julie \\ Marchand, Séverine Marchi, Clémence Pagnoux, Mikaël Pesenti, Alexandre \\ Rabot, Gonca Şenol, Claire Somaglino, Matthieu Vanpeene et Noémi \\ Villars
}

\section{NOTE DE L'AUTEUR}

Année de la campagne : 2020

Numéro et intitulé de l'opération de terrain : 17111 - Taposiris Magna et Plinthine, deux villes de Maréotide

Composition de l'équipe de terrain : L'équipe de terrain était composée de Bérangère Redon (historienne, archéologue et directrice de la mission, CNRS, UMR 5189), MarieFrançoise Boussac (historienne et ancienne directrice de la mission, université Paris Nanterre), Essam Ahmed (archéobotaniste, National Museum of Egyptian civilization NMEC), Charlène Bouchaud (archéobotaniste, CNRS, MNHN), Maël Crépy (géographe et géomorphologue, CNRS, UMR 5189), Louis Dautais (archologue, université Paul-Valéry Montpellier 3, université catholique de Louvain), Sylvain Dhennin (archéologue et égyptologue, CNRS, UMR 5189), Mennat-Allah El Dorry (archéobotaniste, MoTA), Thomas Faucher (numismate, CNRS, UMR 5060 Institut de recherche sur les Archéomatériaux IRAMAT), Paul François (architecte et ingénieur, université de Nantes), Joachim Le Bomin (archéologue, université Paris 1 Panthéon-Sorbonne), Louis Manière (ingénieur au sein du projet Desert Networks (CNRS), spécialiste de SIG), Julie Marchand (céramologue, CNRS, UMR 5189), Séverine Marchi (archéologue, CNRS, UMR 8167), Clémence Pagnoux (archéobotaniste, École française d'Athènes), Mikaël Pesenti (céramologue, Aix-Marseille Université), Alexandre Rabot (spécialiste de SIG, UMR 5189/université Lumière Lyon 2), Gonca şenol (amphorologue, université 
d'Izmir), Claire Somaglino (archéologue et égyptologue, université Paris Sorbonne, UMR 8167), Matthieu Vanpeene (architecte, Ifao/EPHE), Noémi Villars (data manager).

Partenariats institutionnels :

- Ministère du Tourisme et des Antiquités (MoTA)

- Commission des fouilles du Ministère de l'Europe et des affaires étrangères

- UMR 5189 Histoire et Sources des Mondes Antiques HiSoMA

- UMR 8167 Orient \& Méditerranée - Mondes Pharaoniques

- MNHN/UMR 7209 Archéozoologie - Archéobotanique. Sociétés, pratiques et environnements AASPE

Organismes financeurs :

Comme chaque année, la Mission archéologique française de Taposiris Magna et Plinthine (MFTMP) était soutenue par l'Ifao et la commission des fouilles du Ministère de l'Europe et des affaires étrangères (MEAE), ainsi que le CNRS (UMR 5189, UMR 8167 MNHN). La mission a également reçu un important soutien de la fondation Arpamed pour ses travaux sur la vigne (https://www.arpamed.fr/projets/projets-2021/ plinthine/) et un financement extérieur exceptionnel de la part de la foundation Shelby White and Leon Levy dans le cadre du Shelby White and Leon Levy Program for Archaeological Publications (voir le site https://whitelevy.fas.harvard.edu) pour la publication des fouilles du port de Taposiris. Qu'ils en soient tous remerciés.

Données scientifiques produites :

https://taposiris.hypotheses.org/

Les deux sites de Taposiris et Plinthine, en Maréotide, marquent sur la longue durée une frontière occidentale de l'Égypte (fig. 1) : d'abord située à Plinthine, à l'époque pharaonique, celle-ci a basculé vers l'ouest, sans doute à l'époque ptolémaïque, à Taposiris. Alors que la MFTMP s'est concentrée sur les phases les plus récentes et visibles de leur occupation (de l'époque hellénistique à la fin de la période byzantine) depuis sa création, le programme s'efforce depuis 2012 de déterminer la nature des occupations les plus anciennes du site de Plinthine (vestiges d'époque ramesside à saïte), en même temps qu'elle étudie l'impact du nouveau rôle de porte joué par Taposiris à la fin de l'époque romaine/époque byzantine et les conséquences de la conquête arabo-musulmane au vII ${ }^{\mathrm{e}} \mathrm{s}$. sur la ville. En parallèle avec ces interrogations, la mission cherche également à comprendre les mécanismes qui ont conduit au choix des deux sites pour leur implantation. L'étude des activités liées à la viticulture entre pleinement dans ce cadre. 
Fig. 1. Taposiris Magna et Plinthine, aux confins nord-ouest de l'Égypte (B. Redon, fond de carte BingMap).

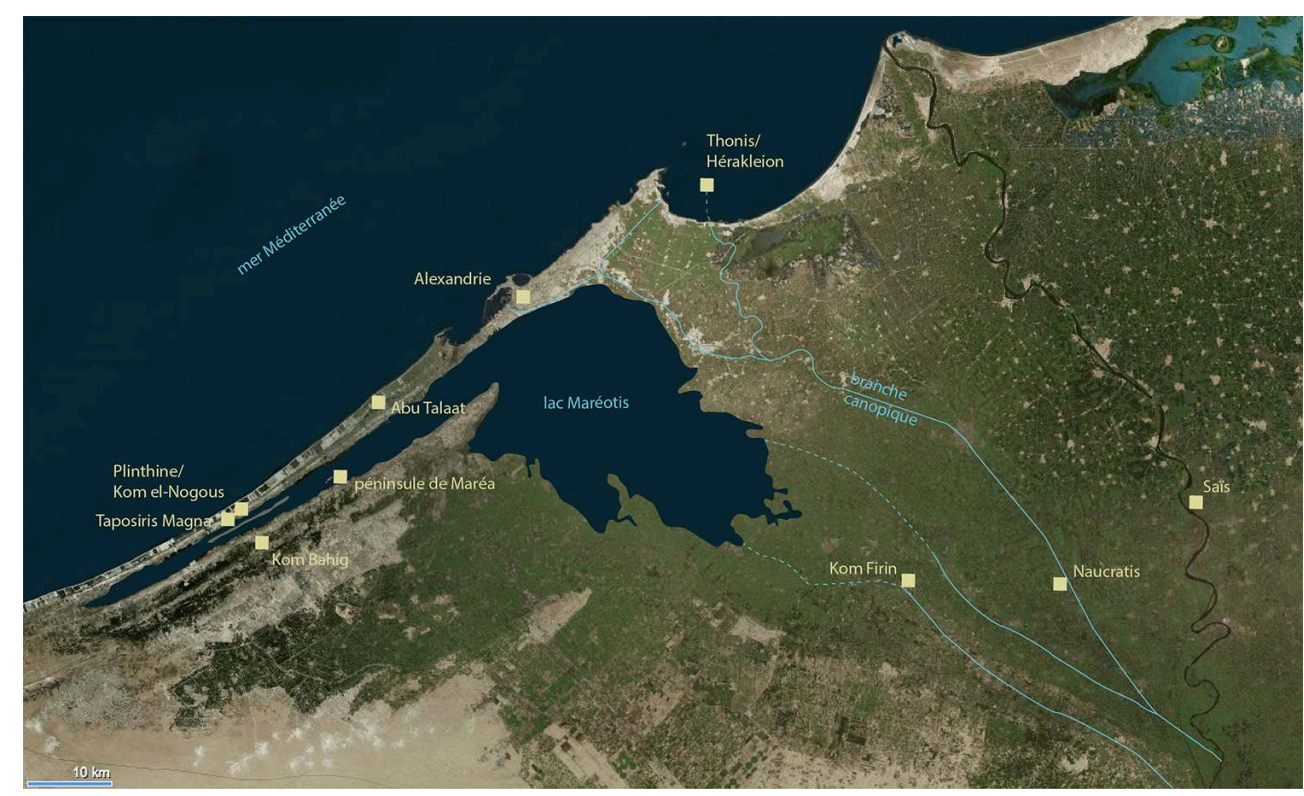

(C) Ifao/MFTMP. 17111_2020_NDMCN_001

2 Grâce au soutien de la commission des fouilles du MEAE, de l'Ifao et du fonds Arpamed, la MFTMP a pris ces dernières années une ampleur inégalée jusque-là, en comptant plus de 25 chercheurs et étudiants parmi ses membres. Dans la même dynamique, la mission a reçu en 2020 le Label Archéologie de l'Académie des inscriptions et belles-lettres, ainsi qu'un substantiel soutien de la fondation Shelby-White pour aider à la publication des fouilles anciennes.

3 En 2020 toutefois, en raison de la crise du Covid-19, les deux missions de terrain programmées au printemps et les missions d'étude au laboratoire d'analyse des matériaux de l'Ifao et dans les magasins de Kôm el-Shougafa et Shallalat à Alexandrie prévues à l'automne ont été annulées. Seule une mission archéobotanique a pu se tenir du 15 septembre au 15 octobre.

4 À la place de ces missions de terrain et d'étude, il a été décidé dès le mois de juin et avec l'accord de nos tutelles de réorienter nos efforts vers la réalisation de travaux en France. Il s'est agi de travaux de post-fouille, avec par exemple la réalisation des catalogues de la céramique traitée sur le terrain à Plinthine depuis 2015, ou encore le travail sur le SIG de la mission et les travaux de modélisation du kôm de Plinthine. Nous avons aussi décidé de travailler de manière intensive à la publication de deux monographies, qui était jusqu'alors en gestation: la première porte sur l'occupation saïto-perse de Plinthine, la seconde sur le port de Taposiris. Pour cette seconde étude, nous avons été aidés par l'obtention d'un prix Shelby-White pour le traitement de nos archives sur le port (fouilles 1998-2010, avec vérifications sur le terrain en 2018-2019) et la publication de la monographie et d'un SIG interactif. Enfin, nous avons poursuivi le travail entamé en 2018 sur les archives numériques de la mission et fait réaliser une base de données intégrée et accessible en ligne. 


\section{Recherches sur Plinthine}

\subsection{Travaux de publication des niveaux saïto-perses}

Louis Dautais, Joachim Le Bomin, Séverine Marchi, Bérangère Redon, Matthieu

Vanpeene

5 À la suite de l'annulation de la mission de terrain, l'équipe qui explore les pentes du kôm de Plinthine, ainsi que les spécialistes qui en étudient le matériel, ont décidé d'entamer la préparation d'une monographie dédiée à la publication de deux quartiers explorés sur le kôm et dont les principales phases d'occupation remontent à l'époque saïto-perse (milieu du viI ${ }^{\mathrm{e}}$ au début du v $\mathrm{v}^{\mathrm{e}}$ s. av. J.-C.).

6 Ces vestiges ont été mis au jour dans et aux abords du kôm (fig. 2), au sud tout d'abord, dans les secteurs 2-nord, 2-sud, 4 et 8 (fouillés de 2013 à 2019), et à l'est, dans le secteur 6 (fouillé de 2016 à 2019).

Fig. 2. Vue générale du kôm de Plinthine (G. Pollin).

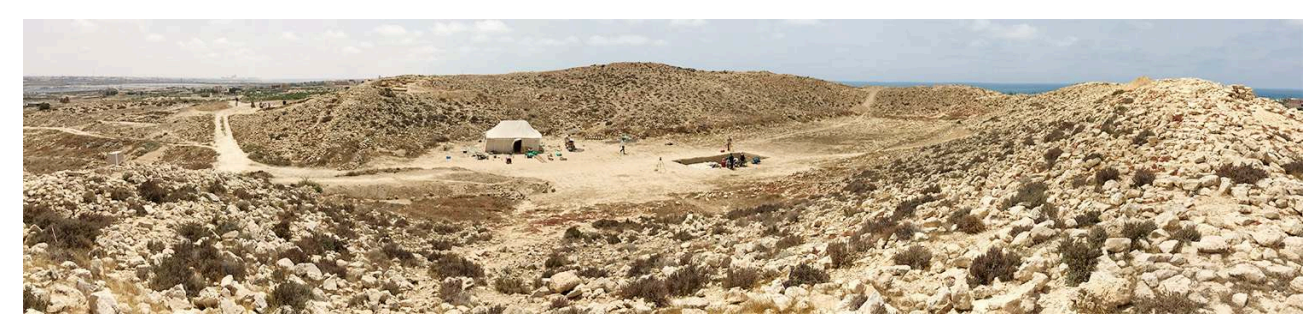

(c) Ifao/MFTMP. 17111_2020_NDMPF_001

7 Le livre permettra de publier ensemble deux quartiers très différents mais contemporains. Le quartier sud est un secteur d'habitat, doté d'unités d'habitation et de stockage, tandis que le secteur oriental abrite aux côtés d'une vaste bâtisse, qui a sans doute servi d'habitation, un fouloir, destiné à la production de vin, puis un édifice de stockage.

8 L'ensemble de la fouille et du matériel (artefacts et écofacts) paraîtront dans le même ouvrage, qui réunira ainsi la totalité de la documentation produite par la mission.

9 Trois réunions de publication ont été organisées en juillet, septembre et octobre 2020. La première, entre Séverine Marchi et Bérangère Redon, a porté sur la publication des PO, ou " petits objets ", une catégorie très large qui réunit toutes les découvertes sauf la céramique, la faune et les restes archéobotaniques. Le mobilier a été classé en catégories et sous-catégories fonctionnelles. Au total, 316 artefacts ont été sélectionnés pour publication.

10 Les deux réunions suivantes se sont tenues à Lyon. Elles étaient destinées à établir le phasage des deux quartiers publiés, phasage qui servira de fondation aux études de matériel (notamment aux typo-chronologies céramiques) et permettra de rédiger les discussions et synthèses en fin d'ouvrage. La réunion du 21 au 23 septembre a réuni Joachim Le Bomin, S. Marchi et B. Redon, avec Paul François le 21 septembre, pour établir un phasage général des secteurs 2, 4 et 8 . Le séminaire de travail sur le secteur 6 s'est tenu le 27 octobre en présence de Louis Dautais, B. Redon et Matthieu Vanpeene. Les diagrammes stratigraphiques des deux zones ont été définitivement validés, ainsi que les plans par phase (fig. 3). 
Fig. 3. Plan des secteurs 2 et 8 de Plinthine durant la phase III (S. Marchi).

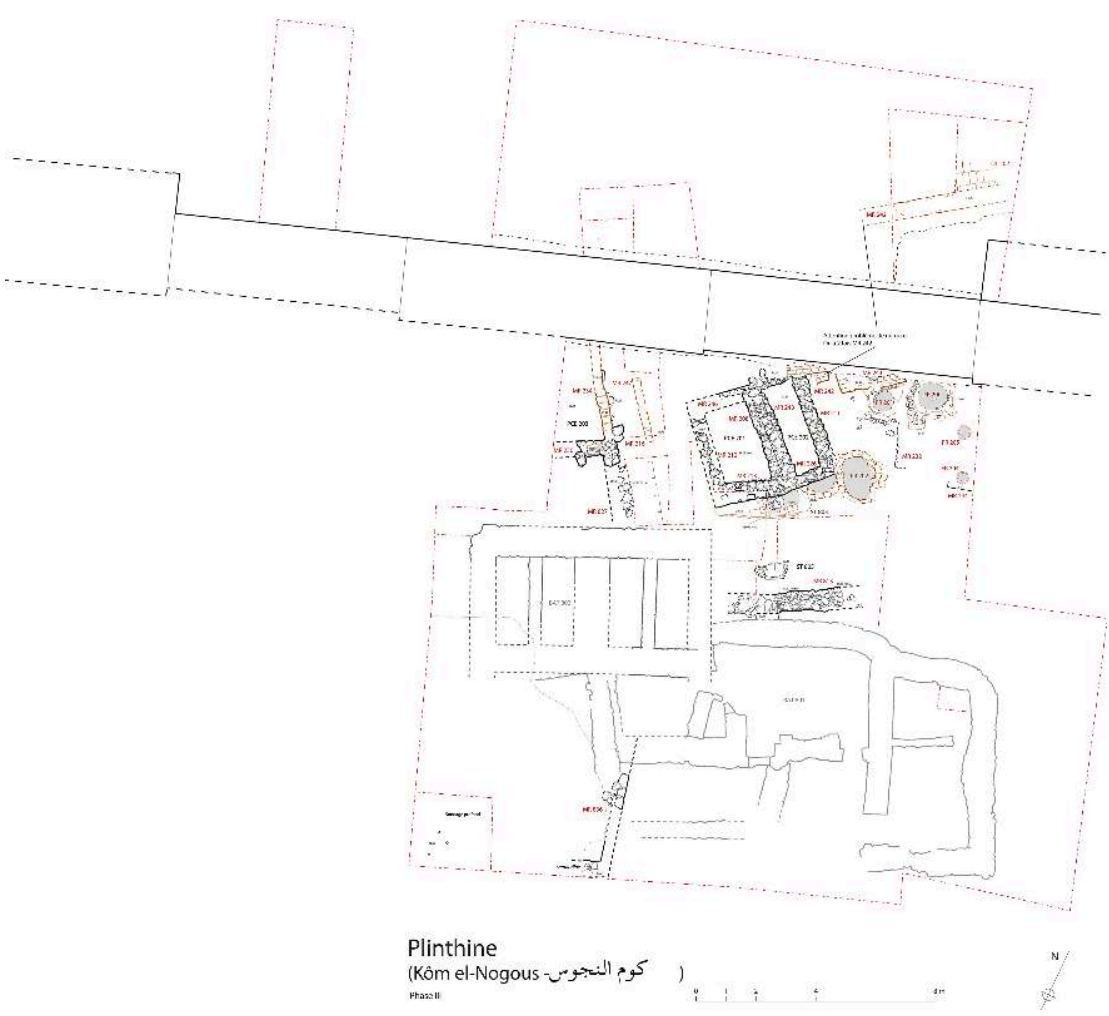

(C) Ifao/MFTMP. 17111_2020_NDMCN_002

\subsection{Travaux de publication sur le temple de Plinthine}

Sylvain Dhennin, Claire Somaglino

Dans le secteur 7, nous nous sommes concentrés sur l'étude et la publication des 17 blocs de réemploi pharaoniques trouvés dans les fondations du temple plus tardif fouillé dans le secteur 7. La plupart appartiennent à un bâtiment cultuel datant du règne de Ramsès II, comme l'indiquent les cartouches du roi inscrits sur certains d'entre eux (fig. 4). D'autres sont issus de monuments privés, ramessides eux-aussi d'après la paléographie et le style, sans doute déposés dans ce temple de Ramsès II. La trouvaille de ces blocs, en pierre locale, permet de compléter la chronologie d'occupation du site, en particulier pour la phase moins bien connue du Nouvel Empire. L'article de Sylvain Dhennin et Claire Somaglino, «A New Temple of Ramesses II on the Western Border of Egypt, on the site of Plinthine », sera soumis au printemps pour le BIFAO 122. 
Fig. 4. Bloc inscrit au nom de Ramsès II mis au jour dans le secteur 7 de Plinthine - PO 675 (a. photographie G. Pollin ; b. dessin et DAO P. François).

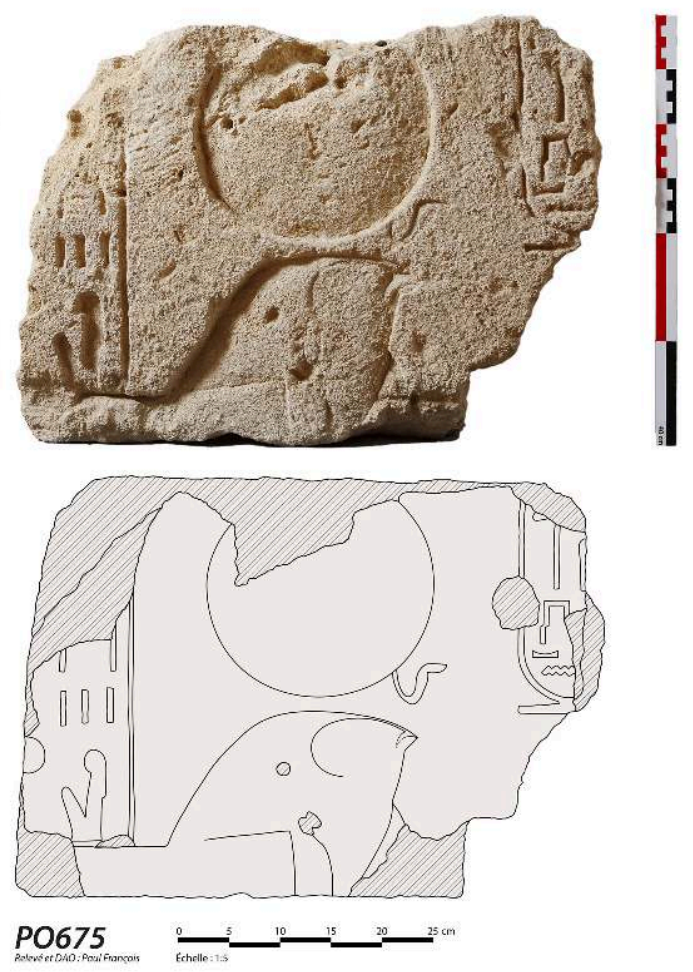

(C) Ifao/MFTMP. 17111_2020_NDMPM_001 et 17111_2020_NDMDM_001

\section{Recherches sur Taposiris : publication de la zone lacustre}

Marie-Françoise Boussac, Maël Crépy, J. Le Bomin, Julie Marchand et al.

\subsection{Obtention du prix Shelby-White : objectifs et plan de publication}

La mission de Taposiris Magna a reçu en 2020 un financement extérieur exceptionnel programmé sur trois ans (2020-2022) dans le cadre du Shelby White and Leon Levy Program for Archaeological Publications. Le projet concerne la zone lacustre de Taposiris Magna (fig. 5), qui a été l'un des premiers dossiers ouverts par la mission. Coordonnée par Marie-Françoise Boussac et B. Redon, cette synthèse porte sur les opérations menées de 1998 à 2010 dans la zone basse du site et sur les vérifications faites depuis 2018 à Taposiris et sur les sites situés plus à l'est, dans les limites de la concession française. 
Fig. 5. Vue générale du port de Taposiris Magna (P. François).

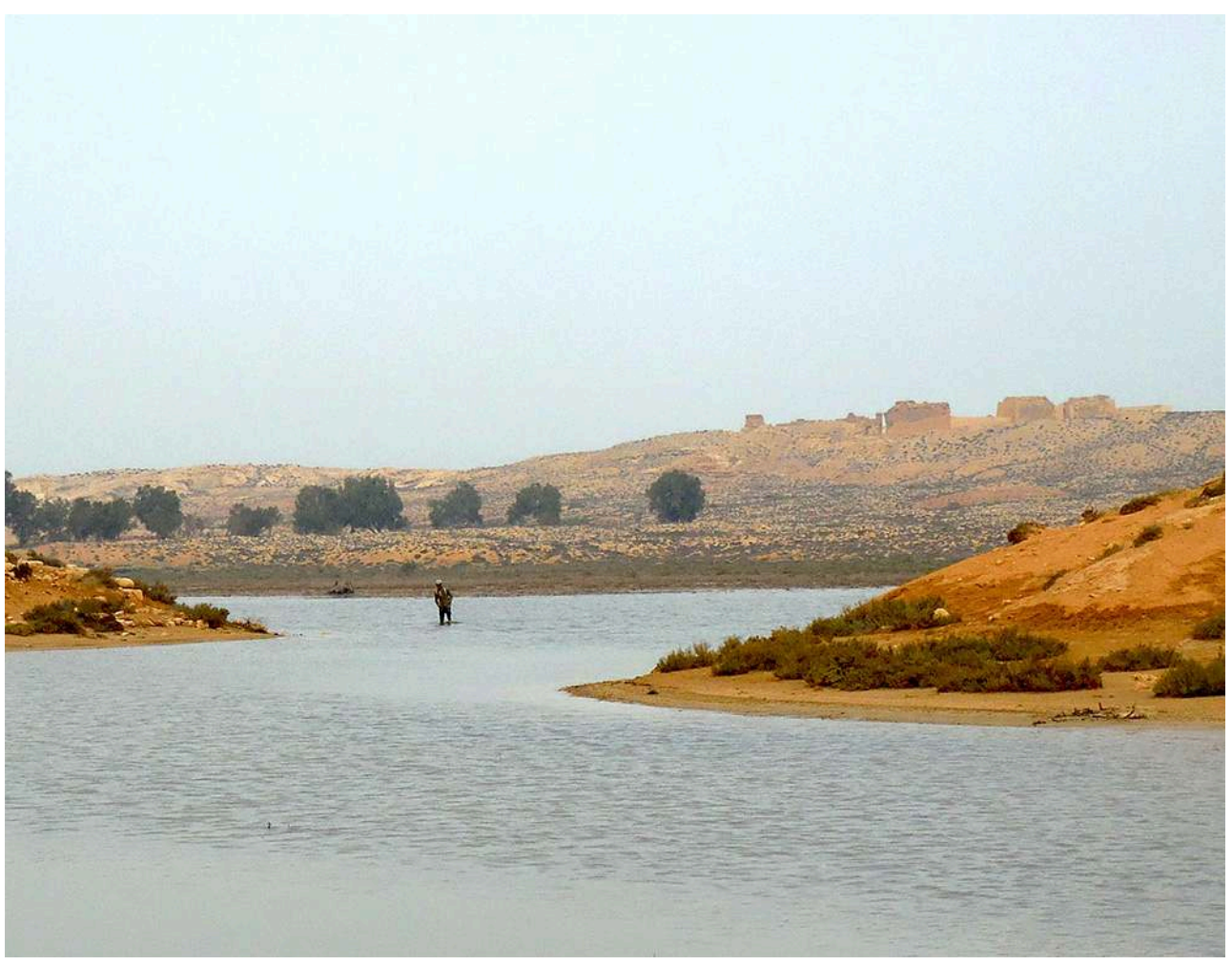

(c) Ifao/MFTMP. 17111_2020_NDMPF_002

Elle comprend les travaux effectués par les nombreux collaborateurs qui sont intervenus depuis le lancement du programme tant sur le terrain que sur l'étude de l'environnement et de la culture matérielle. La coordination de l'étude environnementale sera faite par Maël Crépy, celle des travaux de terrain sera assumée par J. Le Bomin ; celle de la culture matérielle par Julie Marchand.

La publication (qui se présentera sous la forme classique d'une monographie, mais prendra aussi celle d'un Web-SIG) offrira une nouvelle interprétation de l'histoire du lac Maréotis dans l'Antiquité; elle expliquera les changements environnementaux et leur impact sur la dynamique des sociétés et de l'économie. La remise du manuscrit aux presses de l'Ifao est prévue en 2022.

16 Parmi les opérations prévues et effectuées la première année du projet figurent notamment :

- La collecte d'archives scientifiques antérieures (mission Ochsenschlager du Brooklyn College, 1975) et leur traitement numérique. Une correspondance suivie avec les membres de l'expédition du Brooklyn College (Peter Dorman, Carol Bier) a permis de récupérer une série de photos et diapositives, généreusement mises à disposition par ces derniers; les plans de l'architecte Lionel Bier et les carnets d'Edward Ochsenschlager n'ont pour l'instant pas été localisés. Une mission au Brooklyn College est envisagée dès que la situation sanitaire le permettra.

- Après l'établissement de l'état des lieux de la documentation disponible et la conversion numérique des archives argentiques et des diapositives (mission Ochsenschlager, mission française 1998 à 2010 : soit plus de 1600 documents numérisés).

- La mise en harmonie des données (travaux de terrain, études environnementales, études de matériel) et leur renommage selon les normes FAIR en vigueur. 
- Enfin, l'accent étant mis sur les enquêtes environnementales, une mission a été réalisée en 2020 à Paris (BNF et Service Historique de la Défense (SHD), Vincennes) pour retrouver des anciennes descriptions de Taposiris Magna et de son milieu durant l'expédition d'Égypte de Bonaparte, et consulter des cartes essentiellement de la fin du XVIII ${ }^{\mathrm{e}}$ siècle.

Ces avancées à l'échelle régionale ont donné lieu à une publication ${ }^{1}$. Leur application à l'échelle locale a fait l'objet d'une présentation dans le cadre du colloque Riverine harbours organisé par Marine Yoyotte à l'Ifao (15-18 septembre 2019), et une contribution aux actes a été soumise en janvier $2020^{2}$. Enfin, une troisième publication portant sur l'impact environnemental de la mise en place du port au $\mathrm{II}^{\mathrm{e}} \mathrm{s}$. et de ses réaménagements postérieurs a été soumise à la revue Quaternary International ${ }^{3}$.

\subsubsection{Inventaire}

Le tableau ci-dessous résume les données qui seront exploitées dans la future publication de la zone lacustre. Y sont présentées leur nature et leur statut à la rentrée universitaire 2020 :

\begin{tabular}{|c|c|c|c|}
\hline Nature des documents & Type & Zone & Statut \\
\hline $\begin{array}{l}\text { Photos, diapositives et } \\
\text { négatifs }\end{array}$ & $\left|\begin{array}{lrr}\text { Missions } & 1998 \text { à } \\
2005, & 1619 \\
\text { documents } & \text { envoyés }\end{array}\right|$ & $\begin{array}{l}\text { Zones } 1,2,3,4, \\
8,14\end{array}$ & $\begin{array}{l}\text { Numérisé (en } \mathrm{HD} \text { et } \mathrm{SD} \text { ), en } \\
\text { cours de renommage } \\
\text { systématique }\end{array}$ \\
\hline \multirow{6}{*}{$\begin{array}{lr}\text { Archives de } & \text { fouille }: \\
\text { Mobilier : } & \text { céramique, } \\
\text { numismatique, } & \text { verre, } \\
\text { mobilier divers } & \end{array}$} & $\begin{array}{l}\text { Notes, minutes, } \\
\text { relevés, photos }\end{array}$ & $\begin{array}{l}\text { Zones } 1,2,3,4, \\
8,9,15,16,17, \\
18,19, \text { Rahim }\end{array}$ & $\begin{array}{l}\text { Collecte et tri effectués. } \\
\text { Implémentation de la base de } \\
\text { données et DAO en cours }\end{array}$ \\
\hline & \multirow{5}{*}{ Inventaires, dessins } & Zone 1 & $\begin{array}{l}\text { Numérisés, vectorisés, à } \\
\text { étudier }\end{array}$ \\
\hline & & Zone 2 & À étudier \\
\hline & & Zone 3 & $\begin{array}{l}\text { À collecter, à vectoriser et à } \\
\text { étudier }\end{array}$ \\
\hline & & Zone 4 & $\begin{array}{l}\text { Numérisés, vectorisés, en } \\
\text { cours d'étude }\end{array}$ \\
\hline & & $\begin{array}{l}\text { Zone } 16,17,18 \\
\text { et } 19 ; \text { Rahim }\end{array}$ & $\begin{array}{l}\text { Numérisés, vectorisés, études } \\
\text { préliminaires }\end{array}$ \\
\hline
\end{tabular}

TABLEAU 1. Inventaire des archives des fouilles du port de Taposiris.

\subsection{Mission dans les archives parisiennes}

M.-F. Boussac, J. Marchand

\subsubsection{Les archives du Service Historique de la Défense}

19 La mission qui s'est déroulée au SHD (Vincennes, 17 septembre 2020), et à la BNF (département des cartes et plans, 18 septembre 2020), était destinée à étudier les archives liées à l'expédition d'Égypte et diverses cartes du xvIII ${ }^{\mathrm{e}}$ siècle. On sait en effet que l'armée de Bonaparte n'a pu sécuriser la partie occidentale de la Maréotide qu'au 
bout de 3 ans, soit en 1801, ce qui a permis dans un second temps à des savants de l'expédition d'explorer les lieux jusqu'à Abousir (Courier de l'Égypte 106, an IX = 1801), sous escorte militaire, de noter routes, puits, carrières, de relever les ruines et d'étudier le lac. Jusqu'alors les seules observations étaient celles qu'offrait l'atterrage depuis la mer $^{4}$ et ne tenaient compte que des monuments sur la ténia. Le Courier de l'Égypte ( ${ }^{\circ s} 96$, 107) mentionnait brièvement des rapports de reconnaissance militaires particulièrement intéressants pour l'extrémité occidentale du lac: ceux du général Friant, qui s'avança jusqu'à la Tour des Arabes et regrettait son incompétence en matière de ruines, et ceux du chef de brigade J. Cavalier, commandant du corps des dromadaires, chargé de l'ordre dans la région et passionné par les antiquités.

L'objectif était de trouver des rapports plus étoffés de ces opérations. Le bilan est mitigé. Une donation Friant conservée à Vincennes (dossier GR6B76) a livré quelques informations, notamment les Instructions pour la défense d'Alexandrie, qui donnent des renseignements intéressants sur les routes, l'environnement, les points d'eau, les possibilités d'échouage sur la côte.

Un certain nombre de cartes avaient été sélectionnées à partir de l'ouvrage d'A. Godliewska, The Napoleonic Survey of Egypt: A Masterpiece of Cartographic Compilation and Early Nineteenth-century Fieldwork, 1988, ou en discutant avec Lucile Haguet, auteur d'une thèse sur Aegyptus (2007), qui nous a généreusement fourni diverses indications. Parmi les cartes consultées (voir ci-dessous), peu vont à l'ouest du (cap du) Marabout et n'évitent pas toujours (à la différence des cartes de l'expédition d'Égypte) une certaine confusion toponymique : la plus fréquente fait du Marabout la (ou une des) Tour des Arabes. On note également la variété des distances relevées entre Alexandrie et Taposiris ; ce qui pose la question du point de départ des mesures.

Deux cartes tirées des archives de l'expédition d'Égypte se sont révélées particulièrement intéressantes :

- Une carte sur calque (dossier GR6B80) et intitulée « Plan de la côte depuis le marabout jusqu'à la tour des Arabes ( $1 \mathrm{~mm}$ pour cent mètres) ». Elle est peu précise et n'indique aucun nom pour Taposiris dont seules l'« enceinte quarrée » et la "tour des Arabes » sont notées). Plus à l'est l'anonyme kôm de Plinthine est signalé par sa forme arrondie. Le plus intéressant est la mention à l'est de deux sources d'eaux douces avant un troisième plus à l'est d'eaux salées, ainsi que celle de "deux murs " à l'est, qui renvoient à une ancienne route probablement. Malheureusement, la topographie du fond de la vallée de Mariout n'est pas représentée sur cette carte, qui visait surtout à représenter la côte, quelques amers et les points d'eau accessibles rapidement depuis la mer.

- Une carte (dossier GR6MT206C 47), intitulée "Carte du lac Maréotis avec la côte depuis Alexandrie jusques au-delà de la Tour des Arabes » (fig. 6). Le lac ne s'étend pas vers l'ouest au-delà de Plinthine et une très large bande de terre à la hauteur de Taposiris le sépare d'un «marais » à l'ouest. Sur la ténia sont indiqués le temple et la tour. À l'est de ces monuments et à l'ouest de ce qui est indiqué comme un « kom ruiné Aboussir» (= Plinthine) sont dessinées des ruines qui devraient être celles de la nécropole. Rien n'est indiqué pour la ville ou le secteur lacustre. Plus à l'est sont notées près du lac des ruines avec une citerne salée. Ce site est nommé Apis et pourrait être celui de Qoseir ou plutôt de Gamal (distance et attestation d'une citerne non loin du lac actuel). 
Fig. 6. Détail de la « Carte du lac Maréotis avec la côte depuis Alexandrie jusques au-delà de la Tour des Arabes » (Archives de l'Expédition d'Égypte, SDH GR6MT206C 47).

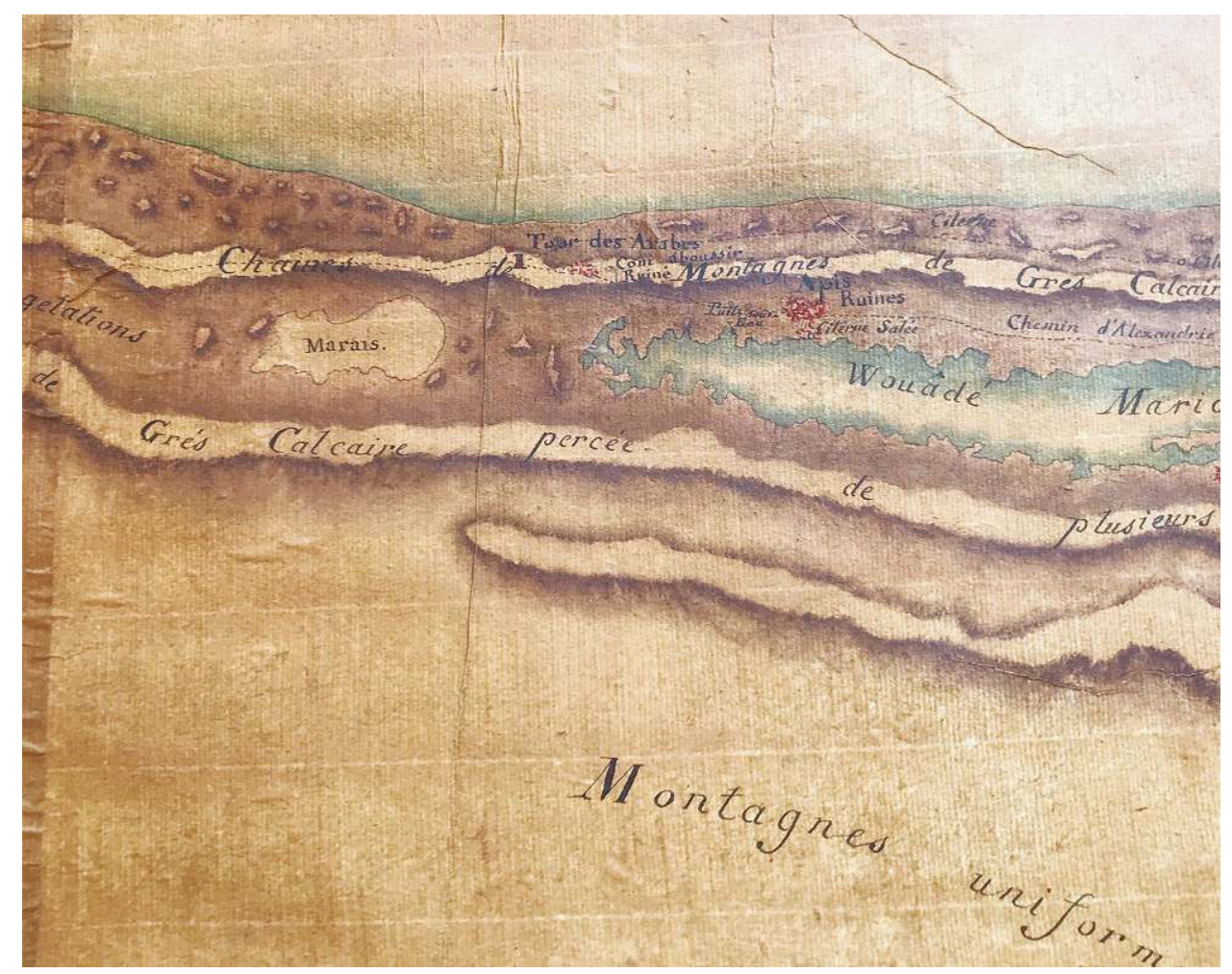

(C) Ifao/MFTMP. 17111_2020_NDMCN_003

Ces deux cartes indiquent comme le général Friant deux chemins entre Alexandrie et la Tour :

- celui du Ouadi Mariout (appelé Darb ou Driah el-Wouade). Il suit depuis l'est le lac jusqu'à la hauteur de Plinthine puis longe la crête jusqu'à la tour (et le temple), évitant la partie basse du site ;

- celui de la mer qui passe sur la crête arrive à la tour (Darh ou Driah el-Barh).

L'itinéraire est commun à l'ouest de la tour, et emprunte le sommet de la ténia.

La donation Friant (Donation Friant, GR6B76) comporte un document manuscrit de la main même de Friant, probablement daté de 1801, évoquant sa mission de reconnaissance jusqu'à la tour des Arabes de Taposiris :

Depuis plus de trois ans on était en Égypte et cependant jusque-là les circonstances n'avaient pas permis de faire reconnaître par terre la tour des Arabes. Le Gal Friant profitant de la tranquillité dans ses provinces [illisible] voulu lui-même dans le mois vendémiaire faire cette reconnaissance. On trouva à 14 lieux sud-ouest d'Alexandrie un grand bâtiment carré construit en pierres calcaires, flanqué de tours et situé sur les bords de la mer. Sa construction, son stile, firent juger qu'il ne devait pas remonter au-delà du $10^{\mathrm{e}}$ siècle si même on pouvait lui donner une origine aussi reculée. On reconnut ensuite la vallée qui conduit de cette tour à Alexandrie et la plage de la tour. On trouva à des distances de deux ou trois lieux dans la vallée, des fontaines ou des mares d'eau douce de très bonne qualité et même des paturages en quantité suffisante dans cette foison pour nourrir de grands troupeaux. Cette reconnaissance ne tarda pas à être profitable car peu de temps après la tribu arabe des houaladalis [Ouled Ali] s'étant révoltée contre les Français en ayant pris sa retraite dans les paturages de cette vallée le gal Frian quitta Alexandrie à la chute 
du jour le 21 frimaire marcha toute la nuit et surprit les Arabes au moment où ils s'y

attendaient le moins. Ils furent battus et perdirent leurs troupeaux.

\section{détaille en bon militaire les éléments stratégiques de la région, en mettant l'accent sur} les points d'eau, la topographie, la nature de la côte :

D’Alexandrie à la tour des Arabes il y a dix grandes lieux depuis le Marabou jusqu'à cette tour. Il y a également six fontaines trois surtout sont assez conséquentes pour abreuver quatre cent cheveaux chaussés il pourrait y en avoir davantage il ne s'agit seulement que de rentrer deux pieds en terre on a de l'eau sur le champ. Le chemin est bon le long de la colline [...]. La chaine de montagnes susdite (sur laquelle est située la tour des Arabes et un ancien monument) du côté du lac mareotis depuis la moitié du chemin d'ici au marabou où elle commence jusqu'à trois lieux de la tour dite des arabes. Dans tout ce qui est escarpé il y a cependant trois communications par lesquelles les troupes pourraient passer sans difficulté de la colline au lac. [...]. Il existe d'ici deux beaux et bons chemins pour aller à la tour susdite. Le premier par le marabou, le second en suivant le lac mareotis. Ce dernier est le meilleur et le plus court (c'est celui que les Arabes prennent pour venir à Alexandrie).

[...]

À partir de la tour des Arabes jusqu'au marabout, la côte est plate et sans écueil sur laquelle un [homme ?] pourrait facilement jetter des chaloupes et opérer un débarquement en hommes et en cheveaux, mais il lui serait très difficile si toutefois il y débarquait des canons et XXXX (quoique de petit calibre) tellement le sable est fin et mouvant.

Environ à soixante pas de la mer on trouve sur toute la crête des rochers [illisible] de dunes jusqu'aux guettes il est vrai qu'elle arrive lorsqu'elle est orageuse; ces roches ne sont pas du tout impraticables, la pierre est tendre et j'ai préféré y passer afin de moins fatiguer les cheveaux qui sur le bord de la mer enfonçaient jusqu'aux jarrets [...].

\subsubsection{Les archives de la BnF}

À la BnF François Mitterrand, grâce à l'obligeance de Catherine Hofmann, conservateur en chef à la Bibliothèque nationale de France, département des Cartes et Plans, nous avons eu accès à un dossier de cartes hors catalogue - Portefeuille 103, division 2, hors catalogue - comprenant essentiellement des cartes de la fin du XVIII ${ }^{e}$ siècle.

Quelques-unes ont retenu notre attention :

- pièce 13B : «Carte de la côte, par le Cne Nouet ", annotée au crayon "d'après Baré, Capitaine de frégate ", 1798, an VI.

Il s'agit de deux cartes superposées, avec en haut, la côte d'Égypte et en bas, le plan du Port Vieux d'Alexandrie. De même que dans d'autres documents de même époque, le Marabout est représenté fermant bien la baie du Port Vieux d'Alexandrie. La tour des Arabes aussi annotée est bien celle de Taposiris, mais elle apparait sur un fait topographique, un cap, qui n'existe pas (à environ 7000 toises du Marabout).

- Pièce 9: «A new plan of Egypt showing the entrances to the Nile etc drawn from the latest authorities » de Heather \& Williams, 1798.

Il s'agit d'une carte sans doute reprise a posteriori car, bien que datée de 1798, elle est annotée d'un texte qui fait allusion à l'expédition de Syrie de 1799. Le «Chersonesus castle », mais aussi une « Arabian tower " ainsi que Plinthine apparaissent bien le long de la côte, quoique très à l'intérieur des terres. Ni la ténia, ni le lac (qui s'arrête à Chersonesos) ne sont représentés au-delà. Un tireté représente les routes prises par les armées françaises entre les branches du Delta jusque dans la région alexandrine. 
Beaucoup de cartes ne sont pas encore numérisées, mais les inventaires papiers indiquent que les réserves conservent plusieurs séries de cartes de prospections et topographiques réalisées depuis 1912. M. Crépy se chargera de leur étude lors d'une prochaine mission afin de compléter le corpus de cartes tant pour l'analyse des sites que pour l'étude de la topographie et de la variation des niveaux lacustres aux XVIII ${ }^{\mathrm{e}}$ et $\mathrm{XIX}^{\mathrm{e}}$ siècles.

\subsection{Reprise des données de fouille}

J. Le Bomin

28 travail de reprise et de mise au net de la documentation des fouilles archéologiques menées depuis 1998 est en cours de réalisation. Celui-ci consiste d'une part à rassembler l'ensemble de la documentation et d'en effectuer l'inventaire et d'autre part à homogénéiser les données selon le système appliqué aux fouilles actuelles. Cette opération consiste notamment à incrémenter les données (photos, relevés de terrain, fiches US, etc.) dans la base de données nouvellement créée par Noémi Villars (voir plus bas). Le retour sur les archives de fouille permet également de vérifier les hypothèses proposées au cours des différentes opérations et au regard des informations acquises plus récemment.

Jusqu'à présent, les archives de fouille se rapportant aux secteurs 1, 3 et 4 (fouilles de 1998 à 2005) ont été rassemblées, triées et homogénéisées. Une partie a d'ores et déjà été intégrée à la base de données, tandis que les diagrammes stratigraphiques et la documentation graphique ont été mis à jour. Pour les opérations plus récentes (secteurs 16 à 19), la majeure partie des données sont enregistrées dans la base.

\subsection{Synthèse et analyses des données environnementales et géoarchéologiques}

M. Crépy

Afin d'évaluer les paramètres (profondeur, étendue, salinité, navigabilité, etc.) du lac à différentes époques, une synthèse des données environnementales disponibles a été initiée par M.Crépy. Elle porte sur six types de données: publications paléoenvironnementales ou géoarchéologiques à l'échelle du Mariout ${ }^{5}$, données non publiées issues des rapports de la mission Taposiris Magna (en particulier celle issues des travaux de Mourad El-Amouri, Jean-Philippe Goiran, Clément Flaux, Hervé Tronchère et Marie-Christine Petipa); images (de 1984 à nos jours) et photographies (programme Corona, de 1965 à 1972) satellitaires, cartes anciennes, contribution de voyageurs, d'explorateurs $\left(\mathrm{XVII}^{\mathrm{e}}-\mathrm{XX}^{\mathrm{e}} \mathrm{s}\right.$.), distribution et chronologie des sites archéologiques publiés dans la région à proximité du $\operatorname{lac}^{6}$. Le croisement d'une partie de ces données ${ }^{7}$ a permis d'envisager plus finement l'évolution du lac entre le IV ${ }^{\mathrm{e}} \mathrm{s}$. av. J.-C. et le viII s. apr. J.-C.

\begin{tabular}{|l|l|l|}
\hline Date & Auteur & Lien \\
\hline 1807 & Arrowsmith & https://gallica.bnf.fr/ark:/12148/btv1b530669569/, consulté le 3 juin 2021. \\
\hline 1818 & Jacotin & https://gallica.bnf.fr/ark:/12148/btv1b531569998/, consulté le 3 juin 2021. \\
\hline
\end{tabular}




\begin{tabular}{|l|l|l|}
\hline 1827 & Coste & https://gallica.bnf.fr/ark:/12148/btv1b8491814n/, consulté le 3 juin 2021. \\
\hline 1850 & St John & https://gallica.bnf.fr/ark:/12148/btv1b53136219p/, consulté le 3 juin 2021. \\
\hline 1866 & al-Falaki & https://gallica.bnf.fr/ark:/12148/btv1b10101071m/, consulté le 3 juin 2021. \\
\hline 1910 & $\begin{array}{l}\text { Survey of } \\
\text { Egypt }\end{array}$ & $\begin{array}{l}\text { https://www.davidrumsey.com/luna/servlet/detail/ } \\
\text { RUMSEY 8 1 317305 90086593:Sheet-47-Bahig, consulté le 3 juin 2021. }\end{array}$ \\
\hline 1942 & $\begin{array}{l}\text { Survey of } \\
\text { Egypt }\end{array}$ & $\begin{array}{l}\text { http://legacy.lib.utexas.edu/maps/ams/egypt/txu-pclmaps-oclc-6559596-el- } \\
\text { hammam.jpg, consulté le 3 juin 2021. }\end{array}$ \\
\hline 1958 & AMS & $\begin{array}{l}\text { http://legacy.lib.utexas.edu/maps/ams/north_africa/txu-oclc-6949452- } \\
\text { nh35-8.jpg, consulté le 3 juin 2021. }\end{array}$ \\
\hline
\end{tabular}

TABLEAU 2. Cartes exploitées au 31/12/2020.

\begin{tabular}{|l|l|}
\hline Date de publication & Auteur \\
\hline 1800 & Rennell \\
\hline 1823 & Le Père \\
\hline 1824 & Jacotin \\
\hline 1825 & Le Père \\
\hline 1829 & Le Père \\
\hline 1829 & Chabrol et Lancret \\
\hline 1849 & St John \\
\hline 1872 & al-Falaki \\
\hline 1885 & Reclus \\
\hline 1935 & De Cosson \\
\hline 1945 & Oliver \\
\hline
\end{tabular}

TABLEAU 3. Contributions de voyageurs, d'explorateurs et d'early scholars dépouillées au 31/12/2020.

\begin{tabular}{|l|l|}
\hline Corona ID & Acquisition \\
\hline DS1016-2088DF007 & $01 / 1965$ \\
\hline DS1109-2171DF010 & $03 / 1970$ \\
\hline DS1111-2167DA024 & $08 / 1970$ \\
\hline
\end{tabular}

TABLEAU 4. Photographies par satellite exploitées au 31/12/2020.

31 La présence de vestiges archéologiques incompatibles (citernes, four à amphores et à chaux en particulier) avec une proximité immédiate au lac, dans le voisinage d'infrastructures de type portuaire ou lacustre, en particulier à l'époque romaine, constituait un paradoxe. L'analyse croisée des données a permis de le résoudre ${ }^{8}$ :

- Le lac Maréotis n'a pas atteint Taposiris durant l'ensemble de l'Antiquité. Il n'était donc pas en hautes eaux à toutes les époques. Certains secteurs étaient au contraire seulement 
marécageux, voire complètement à sec en dehors d'épisodes de précipitations locales hivernales.

- Des vestiges se trouvent actuellement sous le niveau actuel du lac, artificiellement maintenu par la présence de pompes transférant de l'eau entre le lac et la Méditerranée à El-Mexx, près d'Alexandrie.

- Avant la mise en place des pompes, la variabilité saisonnière des niveaux et des étendues du lac était élevée.

- Le fond du ouadi Mariout (vallée occupée par le bras Ouest du lac Mariout et sur le versant nord de laquelle se trouve Taposiris) présente plusieurs seuils topographiques importants, l'un entre Plinthine et l'ouest de Taposiris, l'autre à proximité de Gamal et de l'un des sites de l'île du Mariout (site $23^{9}$ ), et de dépressions marquées qui ont permis la formation de lacs distincts par le passé (le Maréotis saumâtre à l'est de Gamal), et de petits lacs, probablement saisonniers, d'eau douce à l'ouest de Gamal (l'un au sud de Plinthine, l'autre à l'ouest de Taposiris).

- Un canal creusé au $\mathrm{II}^{\mathrm{e}} \mathrm{s}$. apr. J.-C. traverse les principaux seuils topographiques et relie les différentes dépressions entre elles (fig. 7), mettant ainsi en contact les différents corps lacustres et desservant probablement les fours de production d'AE4.

- Les restes de malacofaune contenus dans les curages successifs du canal, à proximité du pont romain, démontrent une salinisation rapide des milieux aquatiques à Taposiris entre le $\mathrm{II}^{\mathrm{e}}$ et le $\mathrm{IV}^{\mathrm{e}} \mathrm{s}$. apr. J.-C., probablement due aux transferts d'eau entre le lac Maréotis, saumâtre et les étendues d'eau douce à proximité de Taposiris, par le canal et le port romain.

- Le niveau de l'eau semble ensuite avoir augmenté dans le courant du IV s. apr. J.-C. et s'être maintenu jusqu'à la fin $\mathrm{du}_{\mathrm{VIII}}{ }^{\mathrm{e}} \mathrm{s}$., avant son assèchement rapide, démontré par $\mathrm{C}$. Flaux (2011). 
Fig. 7. Réseau des canaux navigables du wadi Mariut et ateliers de potier probablement desservis. Photographie Corona datée d'août 1970 (M. Crépy).

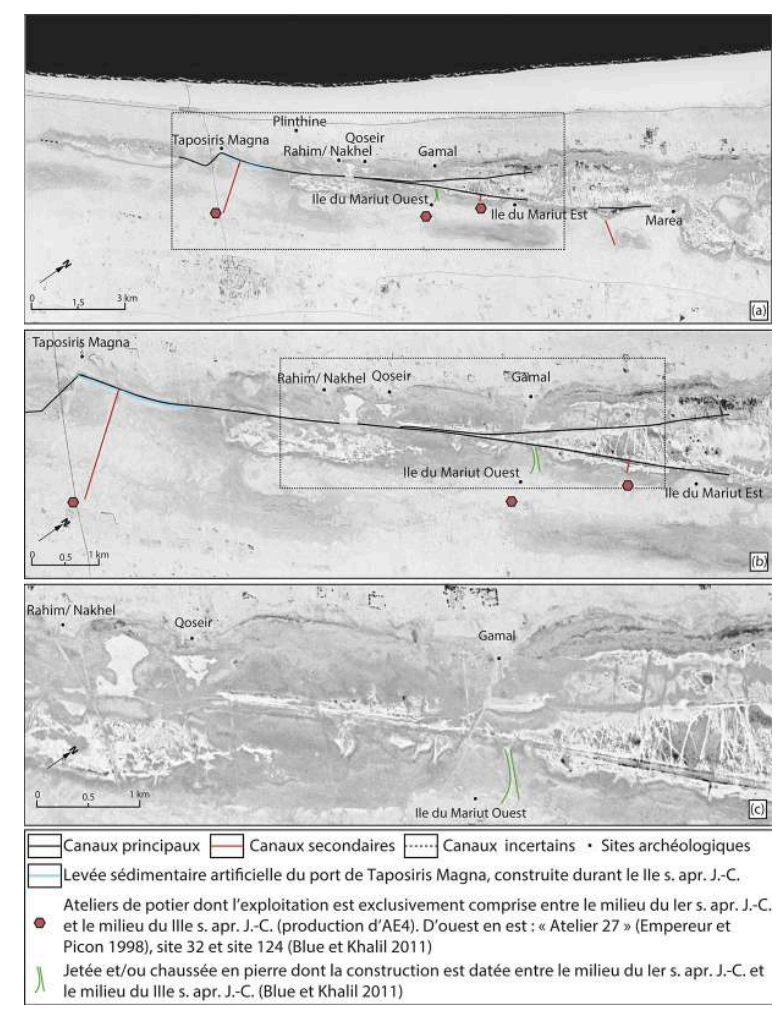

(C) Ifao/MFTMP. 17111_2020_NDMCN_004

Ces données invitent à présent à réévaluer les vestiges à l'échelle du port de Taposiris afin de mieux en saisir le fonctionnement, et de mieux comprendre quelle était la mise en valeur des terrains situés au sud de Taposiris, actuellement sous le lac, mais situés hors d'eau pendant la période hellénistique et une partie de la période romaine. Là où De Cosson (1935) envisageait que le port de Taposiris constituait une mince bande de terre dans un environnement majoritairement lacustre (fig. 8), se trouvaient en fait une majorité de terrains hors d'eau (fig. 9) et un port très circonscrit (fig. 10). Par ailleurs, elles permettront de guider les prospections futures à la recherche des infrastructures lacustres (ou bordières du canal) de Plinthine, Rahim, Gamal et Qoseir, qu'il convient désormais d'envisager comme un complexe portuaire cohérent, plutôt que comme une série de ports ou de jetées indépendants les uns des autres. 
Fig. 8. A. Carte issue de De Cosson 1935, présentant ses hypothèses sur l'étendue du lac et du port durant l'Antiquité. B. Transposition de cette hypothèse sur une image satellitaire de 2019 (M. Crépy, fond de carte Bing Maps).

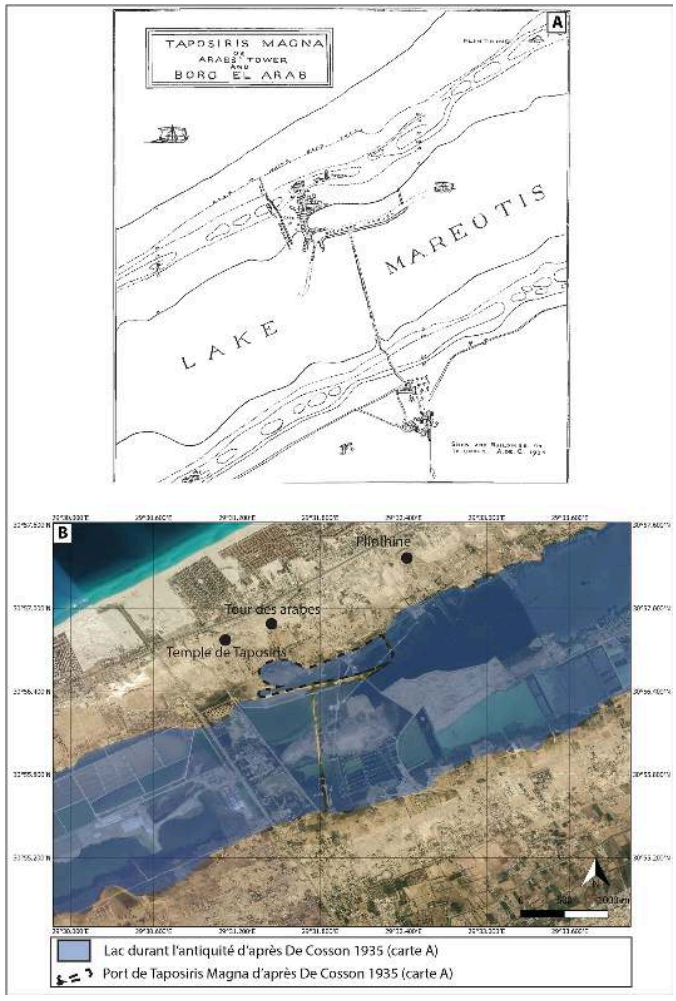

(C) Ifao/MFTMP. 17111_2020_NDMCN_005 
Fig. 9. Carte présentant l'état actuel des hypothèses sur les terres émergées et immergées, sur la formation et le fonctionnement des lacs du ouadi Mariout (M. Crépy, photographie Corona datée d'août 1970).

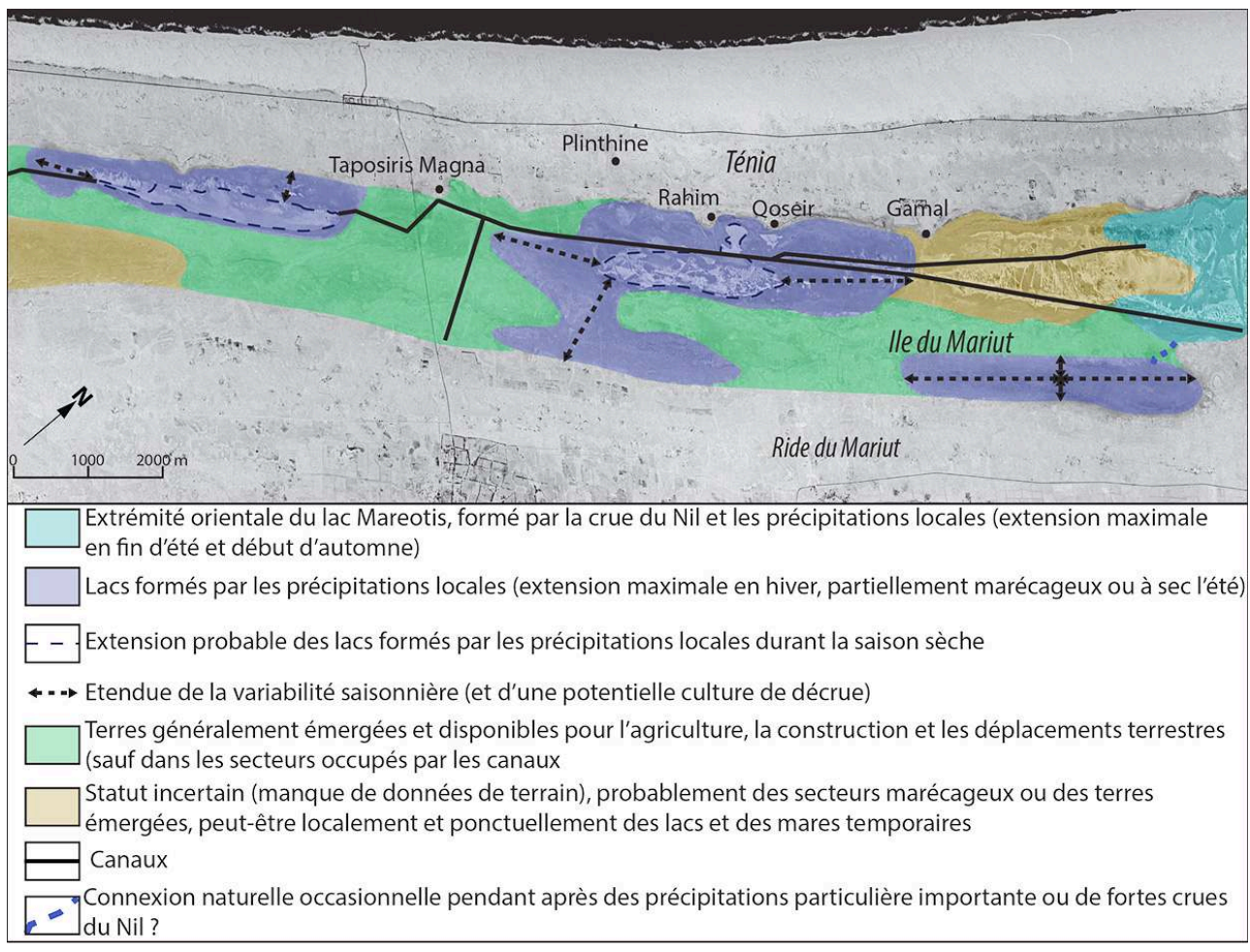

(c) Ifao/MFTMP. 17111_2020_NDMCN_006 
Fig. 10. Carte présentant l'état actuel des hypothèses sur les bassins et les aménagements portuaires à Taposiris Magna (M. Crépy, photographie Corona datée de janvier 1965).

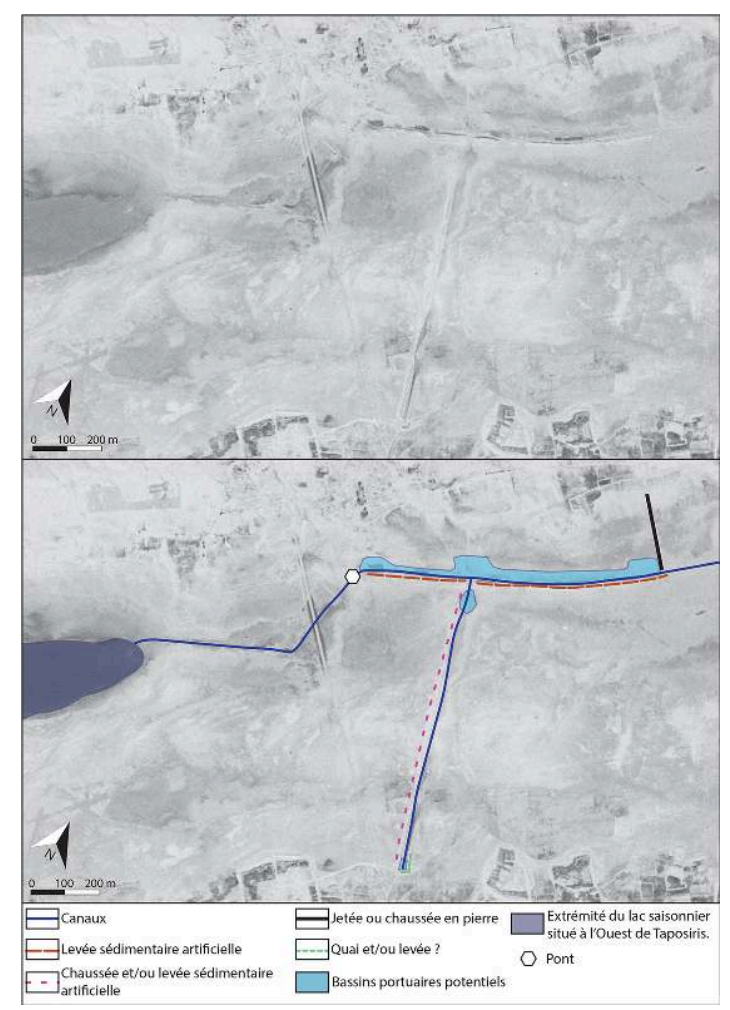

(C) Ifao/MFTMP. 17111_2020_NDMCN_007

\section{3. Études de matériel}

Comme la mission de terrain, les missions d'étude prévues en 2020 ont été perturbées par la crise de la covid-19. Une seule mission d'étude a pu être effectuée en Égypte en 2020 : elle s'est tenue en septembre 2020 au laboratoire d'étude des matériaux de l'Ifao.

Mais plusieurs missions d'étude ont pu être réalisées en France. Ce chapitre comprend ainsi les résultats d'une mission de publication du matériel céramique du secteur 6 de Plinthine. Il est suivi par le rapport sur la publication des anses timbrées du port de Taposiris, conduite durant la première moitié de 2020.

\section{1. Études archéobotaniques}

Charlène Bouchaud, Menat-Allah El Dorry, Clémence Pagnoux, Essam Ahmed

\subsubsection{Introduction}

The archaeobotanical study aims to highlight the ancient use and management of plant resources through time. This report offers an overview of the archaeobotanical work done from November 17 to 27, 2019 and from September 21 to October 15, 2020 at the Laboratoire d'étude des matériaux of the pôle d'Archéométrie de l'Ifao (direction: Anita Quilès, Nadine Mounir). Studied material includes plant macro-remains (seeds, fruits and non-woody vegetative elements) and wood charcoal extracted from soil samples of the French mission of Taposiris Magna and Plinthine. Botanical material is particularly 
rich and well-preserved at Plinthine whereas it is badly preserved at Taposiris Magna. In both sites, desiccated material is almost not preserved because of the humid conditions and the vast majority of the material is charred.

At Plinthine, the material of study includes 120 contexts corresponding to 190 samples (macro-remains or charcoal) and 752.8 liters. The material from Taposiris Magna includes 19 contexts corresponding to 29 samples and 65.1 liters. Data collected in 2011 at Taposiris Magna (Hellenistic baths) are published $^{10}$. We also produced several reports for IFAO and the French Ministry of Europe and Foreign Affairs. Recent results were presented in the last International Congress of Archaeobotany in June, 2019 (IWGP, Lecce, Italy).

All the samples have been processed through dry-sieving or flotation (bucket or machine) during the last laboratory sessions at the IFAO and during the field campaign in May 2019.

We present here only the new results obtained, as well as a summary of qualitative results.

\subsubsection{Macro-remains analysis}

M.-A. El Dorry

A total of 14 samples were studied in 2019 and 28 samples were studied in 2020, covering multiple periods. The study of seeds and fruit this season brought some new elements to the known repertoire of Plinthine.

Flattened barley grains (Hordeum vulgare):

Grains of barley that appear to have been flattened somehow were common in multiple samples.

41 Fruits:

Both grapes (Vitis vinifera L.) and figs (Ficus sp.) are still present widely in the samples.

Weeds and Wild Taxa:

Several new items were observed. However, not all their identifications are secured as some taxa are missing in the reference collection and thus leaving us without a possibility to confirm the identifications. These include Glebionis sp. [from the "daisy family"], Fumaria officinalis [from the "poppy family"], Brassica type (two different ones) [from the "cabbage and mustard family"], Convolvulaceae [the "morning glory family"], and Galium L. [this is a very common contaminant of lentils], Primula sp. [from the "Primrose family"], in addition to several Caryophyllaceae type items (especially desiccated and charred Silene types) [carnation family]. A variety of field weeds particular to cereal cultivation, such as Malva sp., appear frequently in the sample. Suaeda sp. is also very common in the samples.

\subsubsection{Morphometry on grape pips}

C. Pagnoux

Morphometric analyses of grape seeds from Plinthine aim to shed light on the past grapevine diversity and its evolution through time on this site (fig. 11). We use geometric morphometry to quantify morphological diversity. 
Fig. 11. Location of the archaeological remains associated with viticulture at Plinthine (M. Vanpeene).

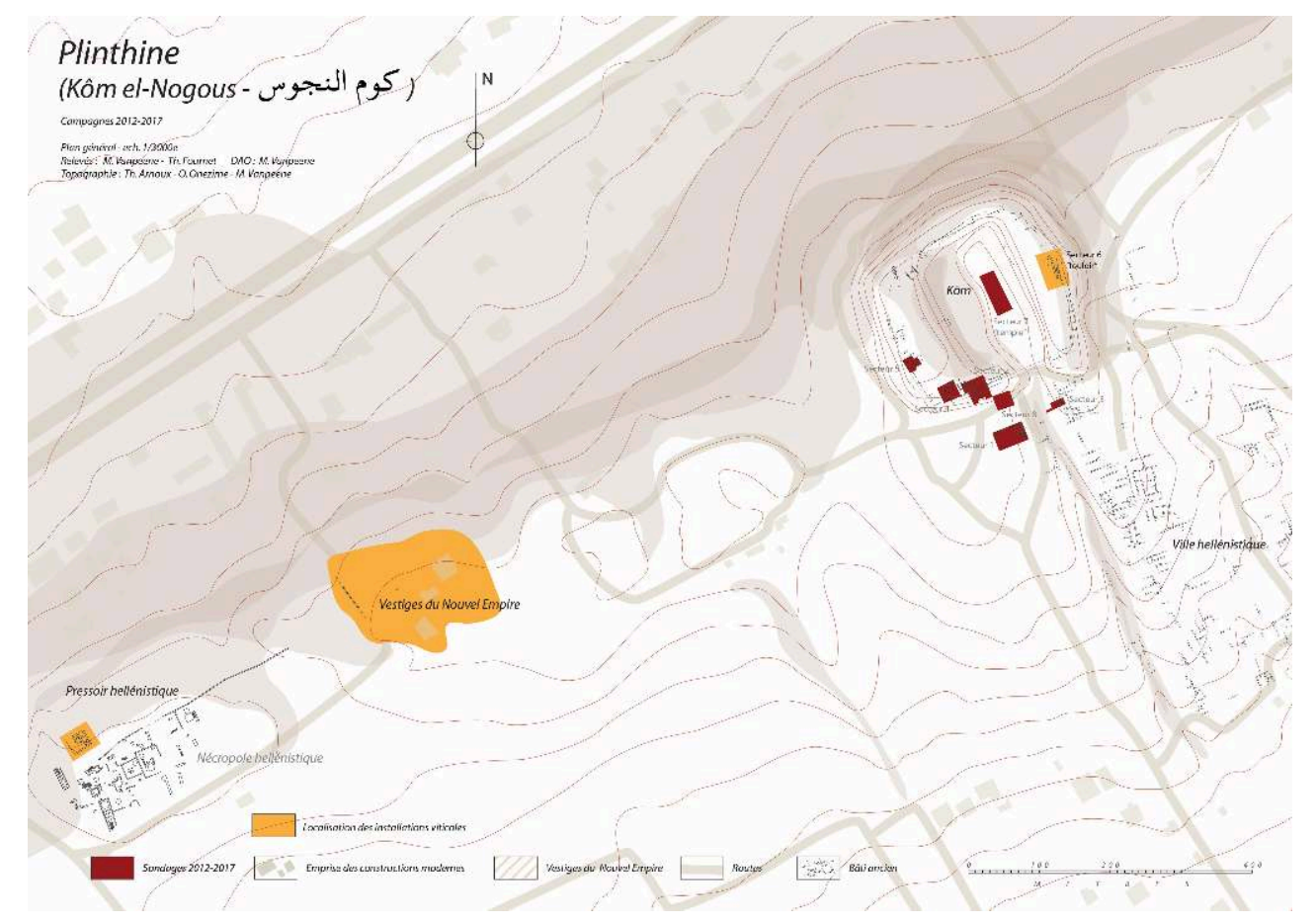

(c) Ifao/MFTMP. 17111_2020_NDMCN_008

The first laboratory mission conducted in September 2018 aimed to photograph a first series of grape pips from Plinthine. Then, most of these pips have been analysed in 2019 and provided interesting results. In order to enlarge our knowledge of the grapevine diversity at Plinthine, this second laboratory mission was dedicated to new morphometric analyses on grape pips discovered among the samples that have been floated and sorted in 2019.

Grape pips from five samples from Plinthine and one sample from Taposiris Magna were selected on the basis of their chronology. We have chosen samples dated to the Saite period (7th/6th c. BC) and to the early Persian period (5th c. BC) which were not well represented in the analyses conducted in 2018-2019. Since material from sector 6 is very abundant, large grape concentrations were favoured, as far as possible.

6 Samples with grape seeds dated to the Ptolemaic period are scarce. In addition to the material already studied, one single sample from Taposiris Magna dated to that period contained at least 10 pips. Eventually, only 7 were enough well preserved to be analysed.

47 A very rich sample was chosen in order to test morphological diversity within a sample. The material from US 6314 (389 pips analysed in 2018 and 301 pips photographed in 2019) will allow us to test whether the number of identified morphotypes increased with the number of analysed pips. Until now, morphogeometric analyses on grape pips never took into account such a large amount of seed belonging to one single context.

686 archaeological grape pips have been photographed in dorsal and lateral view using a camera connected to a computer via USB port (Dino Lite). The pictures have been converted into black silhouette on a white background using Photoshop software. 
outline of the pips will be extracted and decomposed in a series of trigonometric function called harmonic, using the Elliptic Fourier Transform method (EFT). ${ }^{11}$ The coefficients of the harmonics will be used in statistical analyses (Principal Component Analyses, Linear Discriminant Analyses) as shape descriptors. The grape pips from Plinthine will be compared among them, and with material from Ptolemaic layers at Karnak, Egypt and with material from Greece dated to the Archaic (7th-6th c. BC) and Hellenistic period (3rd-2nd c. BC). In order to identify morphotypes (varieties or group of varieties), the grape seeds from Plinthine will be compared to a modern reference collection.

\subsubsection{Charcoal analysis}

C. Bouchaud

Charcoal analysis brings information on fuel selection and environment. During the laboratory session, we analyzed six new samples. Two of them come from Plinthine, sector 6, dated to 7th-5th c. BCE. We also studied all the samples containing charcoal from Taposiris Magna, i.e. sector 13 dated to Late Antique periods 5th-7th c. AD and sector 19 dated to Roman period, 2nd and 3rd c. CE.

\subsubsection{Plinthine results}

51 The analysis of the two samples from sector 6 (6345_Bot1, 6349_Bot1) at Plinthine provide results similar to those already obtained to date on sectors 6 and 8 . Shrubs from the Amaranthaceae family and Faboideae subfamily, as well as grapevine wood dominate. Tamarisk, fig tree, capper tree and cf. Leptadenia pyrotechnica and possible date palm trunk are recorded as well. These taxa illustrate part of the spontaneous shrubby (Amaranthaceae, Faboideae, capper tree, cf. Leptadenia pyrotechnica) vegetation growing in the neighboring during the Third Intermediate Period and Saite/Persian periods and indicate salty and xeric environment. The discoveries of grapevine and fig tree charcoal show that the two species were locally cultivated, maybe associated with a few date palms. Tamarisk represents either spontaneous shrub/tree or maintained plant, used for the wood or as barrier hedges to protect crops from wind and sand for instance (PlantUse Français contributors 2015). The tree is also known to be welladapted to brackish lands and edged of marshes and salt lakes where it depollutes soils and makes the lands suitable for cultivation. ${ }^{12}$

Beside these results that confirm the homogeneity of the charcoal corpus at Plinthine, four new taxa are recognized among the two samples: Acacia sp., Diplotaxis type, Fagus orientalis/sylvatica (oriental/common beech), and Lycium sp.

\subsubsection{Taposiris Magna results}

The first study lead on samples took during the 2011 campaign already showed the use of reed (Arundo/Phragmites) and other monocotyledons, as well as Amaranthaceae shrubs and tamarisk for feeding the Ptolemaic Baths..$^{13}$ Charcoal out of abandonment layers of Ptolemaic baths correspond to reeds, indeterminate monocots, date palm midrib, Nile acacia, Faboideae, Pinus nigra/sylvestris (black/scot pine), Pinus halepensis/ pinea (Aleppo/maritime/umbrella pine), poplar/willow (Populus/Salix) and tamarisk. The study of new samples groups taxa already identified in the old samples of Taposiris Magna (Acacia, Amaranthaceae, reed, Faboideae, the two pine groups). Grapevine

Bulletin archéologique des Écoles françaises à l'étranger , Égypte 
charcoal is also attested for the first time at Taposiris Magna in a few quantities in a Roman layer (19005_Bot1). The new study also increases the number of taxa: Cupressus/ Juniperus, (cypress/juniper) sometimes identified to species rank (cypress), Olea europaea (olive tree), Punica granatum (pomegranate tree) and Quercus sp. (deciduous oak).

New data obtained at Taposiris Magna are very exciting. They show the presence of a large diversity of wood taxa. Some likely naturally grew in the neighbourhood (such as acacia, Amaranthaceae, reeds, Faboideae) and indicate xeric/marshes environment. The presence of potential locally cultivated fruits - grapevine, olive tree and pomegranate tree - is tricky to interpret so far: do they correspond to elements grown on or near the site - although soils around Taposiris Magna are not as suitable for grapevine as the slopes around Plinthine - or do they come from Plinthine (or elsewhere)? The presence of foreign taxa - cypress, two species of pines and oak - is also difficult to consider. As for beech found at Plinthine, we hardly believe that such wood was imported for fuel consumption. Since they are all wood of high quality known for building purposes, they do correspond to re-use of wood. All the imported species are high quality wood recognized and commonly used as timber. We must consider that part of them could correspond to worn out shipbuilding timber components.

\subsection{Mission de publication du matériel céramique de Plinthine}

Mikaël Pesenti

Le travail a été effectué pendant le mois de juillet 2020. Il a consisté à stabiliser les données, à faire le point sur ce qu'il restait à traiter, à mettre au net la documentation graphique et à rédiger un premier chapitre comprenant une introduction à l'étude qui sera publiée dans la monographie en préparation sur les niveaux saïtes de Plinthine (voir plus haut).

\subsubsection{Inventaire et mise au net des données}

Pour l'heure, nous dénombrons sur l'ensemble des quatre secteurs 35337 NR pour 3872 NMI céramiques. Sur sept campagnes de fouilles (2013 à 2019), 800 minutes ont été réalisées. La mise au net de la totalité des dessins a été faite en juillet 2020. Nous disposons ainsi de 1817 dessins de céramique provenant des secteurs 2-nord, 2-sud, 6 et 8 (fig. 12).

Au terme de ce travail, il s'avère qu'un nombre important d'US (208 exactement sur l'ensemble des secteurs 2-nord, 2-sud, 6 et 8, dont une trentaine sont jugées prioritaires) n'ont pu être intégralement documentées pendant les campagnes de fouilles. Une mission d'étude est prévue au début de 2021 pour compléter les données en vue de la publication monographique. 
Fig. 12. Matériel céramique de six US de la phase B du secteur 6 (M. Pesenti).

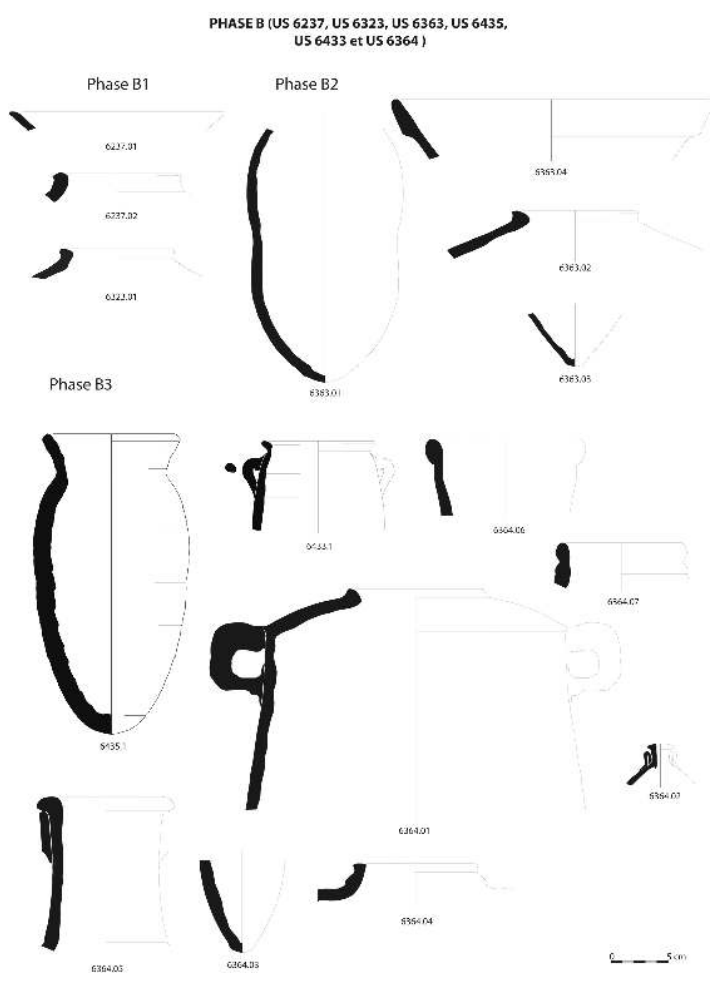

(c) Ifao/MFTMP. 17111_2020_NDMDM_002

\subsubsection{Introduction à l'étude de la céramique de Plinthine}

Le site de Plinthine livre un témoignage important sur une ville égyptienne sur le littoral méditerranéen. À travers l'étude de quatre secteurs (secteur 2-Nord, secteur 2Sud, secteur 6 et secteur 8) densément occupés aux époques saïte et perse sont mis en évidence des pans très différents de la société égyptienne. Même si des traces d'une occupation plus ancienne remontant au moins à la Troisième Période intermédiaire sont avérées, la documentation céramique s'étend majoritairement de la fin $\mathrm{du} \mathrm{VII}^{\mathrm{e}}$ jusqu'à la fin du $v^{e} s$. av. J.-C. Certes une occupation ptolémaïque importante - dont témoignent quelques structures à l'instar du mur massif MR 201 ou encore un long drain dans les secteurs 2 et 8 - suggère une certaine continuité de l'occupation. Cependant, les vestiges de l'époque ptolémaïque sont peu nombreux et même les premières couches de surface livrent avant tout du mobilier céramique daté de l'époque saïte ou perse. Les secteurs 2-nord, 2-sud, 6 et 8 ont tous cette particularité.

Les données céramiques permettent de mettre en lumière plusieurs aspects de la ville de Plinthine aux époques saïte et perse. La question de l'identité des habitants implique de s'attarder sur les éventuels et problématiques critères de reconnaissance à partir du vaisselier, des habitudes de consommation ou même de production. En effet, une partie des habitants de Plinthine produit du vin (voir le fouloir et l'étude archéobotanique), mais les amphores retrouvées sur le site attestent également qu'ils en importent massivement depuis la Grèce ou le Levant. Or les secteurs fouillés sont constitués de contextes domestiques et artisanaux égyptiens. L'apport de céramique importée (conteneurs principalement mais également de la vaisselle fine) du monde grec, 
levantin ou chypriote y est considérable et la présence de cette céramique dans des habitats relativement simples doit être questionnée (à la fois sur l'identité des consommateurs du vin que les amphores contenaient, l'identité des habitants du village de Plinthine, et sur la question aussi du remploi éventuel des amphores à Plinthine).

\section{3. Étude des anses timbrées}

Gonca şenol catalogue des 98 anses timbrées trouvées dans les trois secteurs situés dans la partie occidentale du port. Ils sont presque tous trouvés en position secondaire, dans les couches de dragage du chenal artificiel, mais révèlent que le complexe d'époque impériale (première moitié $\mathrm{du} \mathrm{II}{ }^{\mathrm{e}} \mathrm{s}$. apr. J.-C.) a bouleversé une zone occupée à l'époque hellénistique, entre le milieu du $\mathrm{II}^{\mathrm{e}} \mathrm{s}$. av. J.-C. et le $\mathrm{I}^{\mathrm{er}} \mathrm{s}$. av. J.-C. et abritant des bâtiments de stockage proches du secteur 3 fouillé entre 2001 et 2005 sur la levée artificielle de direction E/O. Les premières conclusions montrent que l'origine des amphores correspond à ce que l'on trouve à Alexandrie : essentiellement du vin de Rhodes, suivi par du vin de Cnide. Le catalogue et les premières conclusions seront complétés par une étude comparative avec la situation que l'on observe dans la ville haute (terrasse Breccia) ; une version préliminaire a déjà été rédigée.

\section{Outils numériques pour l'enregistrement, la modélisation et l'archivage des données}

\subsection{Développement d'une base de données pour la fouille MFFTM}

N. Villars

Il a été décidé de créer en 2020 une nouvelle base de données avec le logiciel FileMaker, vouée à remplacer les bases existantes mais vieillies de Taposiris et de Plinthine, afin qu'elle reflète les besoins des chercheurs. Celle-ci a pour but d'intégrer les résultats de différents domaines d'études: archéologie; archéozoologie, archéobotanique, céramologie, artefacts, documentation photographique, illustrations (plans, coupes, etc.).

Les bases préexistantes présentent plusieurs problèmes qu'il a fallu contourner :

- plusieurs bases indépendantes les unes des autres ;

- aucun accès à la gestion des bases de données (pas de code d'accès disponibles) ;

- structure créée sous FileMaker 5 en 1989, devenue totalement obsolète.

La seconde étape, après la conception de la base, a consisté à exporter les données existantes de la base de données de Taposiris dans douze nouvelles tables non relationnelles, afin de les épurer et de comprendre leur interdépendance. La troisième étape a consisté en l'importation des données existantes dans la nouvelle base de données. Celle-ci a été réalisée à l'hiver 2020/2021. La base est désormais accessible à tous les membres de l'équipe sur un serveur Filemaker mis à disposition par HumaNum. Elle sera dorénavant incrémentée directement sur le terrain en 2021, grâce à l'achat de tablettes numériques par le laboratoire HiSoMA. Elle servira aussi à la

Bulletin archéologique des Écoles françaises à l'étranger , Égypte 
publication des secteurs du port fouillés anciennement, puisque les données de ces travaux vont y être intégrées rétrospectivement.

\subsection{Archives numériques de la mission}

64 Dans le cadre du projet financé par Shelby-White, mais plus largement dans la continuité des efforts entrepris depuis quelques années pour ordonner et rendre accessible nos archives, un travail de classement a encore été fait en 2020 sur les archives numériques de la mission Taposiris-Plinthine, sous l'impulsion de M.F. Boussac, J. Le Bomin et J. Marchand.

\subsection{SIG et acquisition d'images satellites et réalisation d'un MNT}

L. Manière l'aide de 300 points échantillonnés aléatoirement. On observe des écarts absolus de $3.6 \mathrm{~m}$ pour $80 \%$ des valeurs. Les écarts les plus élevés se trouvent dans les secteurs de faibles contrastes (comme les zones d'extraction de l'ouest et du nord-ouest ou les espaces sableux du sud-est), à la bordure avec la mer (différente de trait de côte entre le SRTM et le MNT) ainsi qu'au niveau de la lagune (dépendant du niveau de l'eau à l'acquisition des données des images et du SRTM) (fig. 13). 
Fig. 13. Qualité altimétrique du MNT de Taposiris et Plinthine par comparaison au SRTM (L. Manière).

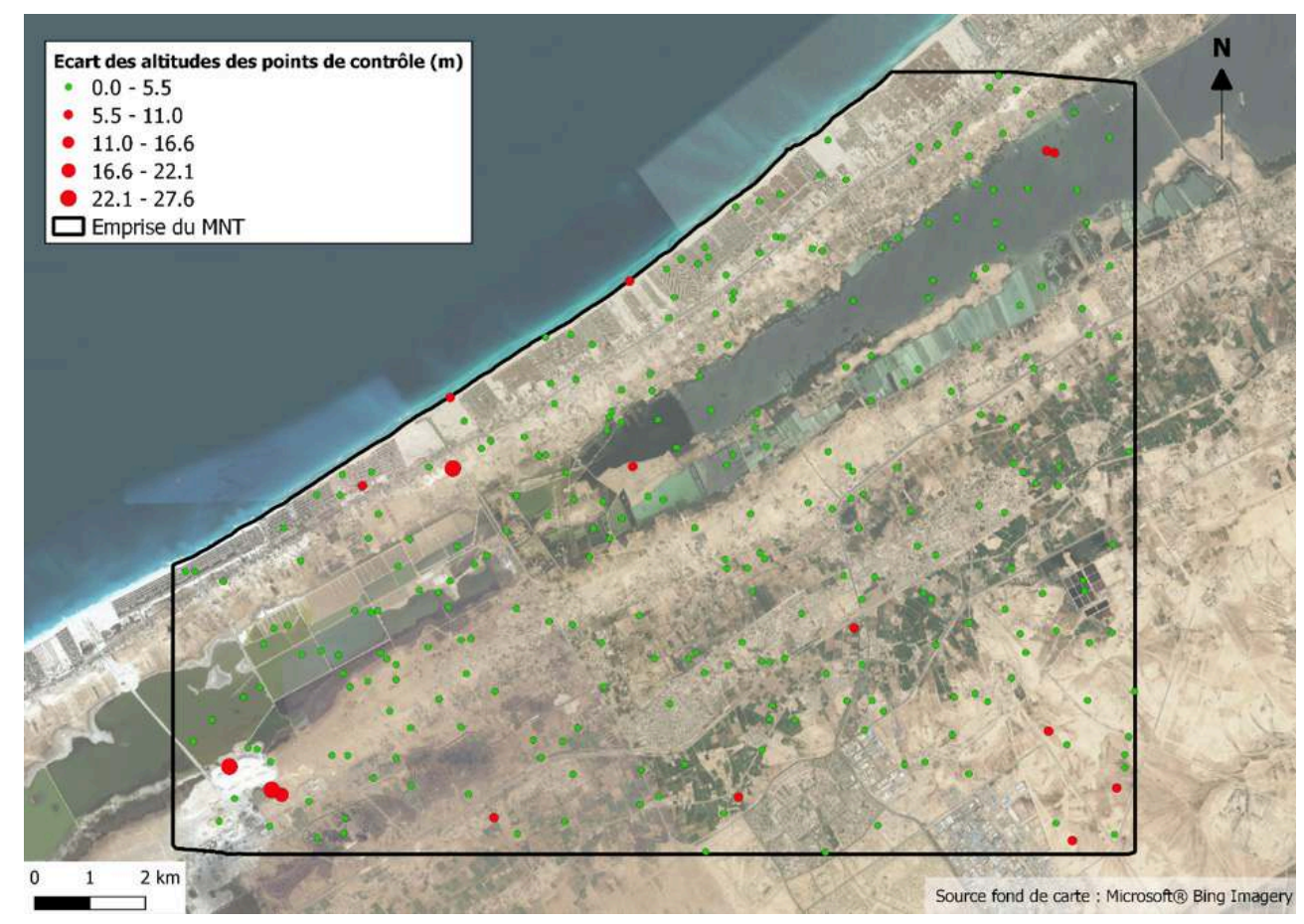

(c) Ifao/MFTMP. 17111_2020_NDMCN_009 pour l'Afrique de $5.6 \mathrm{~m}$ en verticale absolue et de $11.9 \mathrm{~m}$ en horizontale ${ }^{14}$. La précision verticale du MNT produit ne peut donc pas être supérieure, il comprend les mêmes incertitudes mais a une résolution 30 fois supérieure. La précision horizontale est très améliorée à l'aide de l'image Bing haute résolution qui a servi à la calibration.

\subsection{Modélisation}

Paul François

Faute d'accès au site pour cette année 2020, les travaux concernant la restitution archéologique du kôm de Plinthine se sont portés sur la capitalisation des données précédemment acquises et sur la préparation des futurs travaux.

71 Nous avons tout d'abord rendu possible l'accès à la restitution du fouloir saïte de Plinthine au plus grand nombre en le déposant sur une plateforme libre d'accès. Le modèle, conçu en concertation avec les archéologues grâce à l'acquisition des données du secteur 6 lors des saisons 2018 et 2019, était jusqu'à présent uniquement visible sous la forme d'images statiques en deux dimensions. Le dépôt sur la plateforme SketchFab ${ }^{15}$ autorise non seulement le déplacement à l'intérieur du modèle depuis n'importe quel ordinateur connecté à Internet, mais il permet aussi d'obtenir des informations sur le fonctionnement du fouloir et les choix effectués pour sa restitution. Il s'agit donc d'un dispositif de médiation, qui a pu, en période de crise sanitaire, suppléer à l'impossibilité de faire visiter cet espace avec des dispositifs de réalité virtuelle (casque de réalité virtuelle, notamment), tout en le rendant accessible au plus grand nombre. 

(secteur 10), effectué en 2020, a également fait l'objet de la production d'un modèle 3D et d'une phase de préparation permettant d'envisager une restitution en trois dimensions de son fonctionnement. Enfin, la documentation archéologique et les relevés photogrammétriques produits depuis 2016 concernant le secteur 8 (au sudouest du kôm), ont été rassemblés et ont fait l'objet d'une analyse conjointe avec les archéologues en vue de la restitution d'une partie du secteur, autour d'une des habitations fouillées. Tant le secteur 10 que le secteur 8 pourront donc faire l'objet d'une restitution lors de la saison prochaine.

Ces restitutions s'inscrivent dans un projet plus vaste de modélisation de l'ensemble du kôm, qui avait déjà motivé ma présence lors de la mission 2019. En 2020, l'achat de trois images satellites Pleiades de 2016 se chevauchant au niveau du kôm a permis la réalisation, en utilisant la photogrammétrie, d'un Modèle Numérique de Terrain à grande échelle (voir plus haut). Ce document permettra de modéliser et mieux comprendre l'implantation du site de Plinthine sur la ténia entre le lac et la mer Méditerranée, et ce en trois dimensions.

74 À partir du modèle numérique de terrain, nous avons extrait une «tranche » de ce territoire pour en produire un modèle 3D (fig. 14). L'enjeu de la restitution de cette tranche de territoire autour du kôm de Plinthine est multiple et révèle le télescopage d'échelles qui fait l'intérêt du projet et sa complexité. Il s'agit non seulement de représenter les différentes structures géologiques qui font la particularité de ce territoire, mais également l'environnement agricole (permettant la production de vin), urbain, et micro-local des différentes restituions envisagées (les deux fouloirs, le secteur 8). Les différentes époques des vestiges restitués imposent en outre une approche diachronique de l'environnement paysager et urbain.

Fig. 14. Début de modélisation du kôm de Plinthine à partir du MNT (P. François).

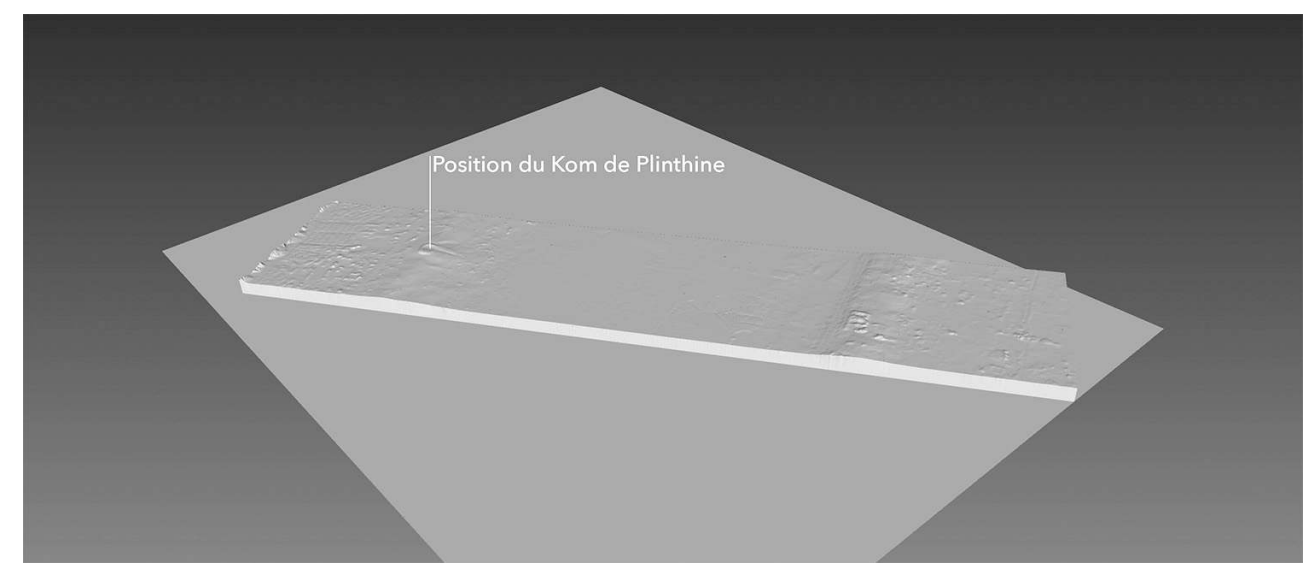

(C) Ifao/MFTMP. 17111_2020_NDMCN_010

Bulletin archéologique des Écoles françaises à l'étranger , Égypte 


\section{Liste des publications, conférences et autres actions de valorisation réalisées en $\mathbf{2 0 2 0}$}

\subsection{Monographies/ouvrages collectifs}

1. Marie-Françoise Boussac, Olivier Callot, Patrice Georges-Zimmermann (éd.), avec des contributions de Gonca Cankardeş-şenol, Maël Crépy, Thomas Faucher, Camélia Georges, Cécile Harlaut, Dominique Kassab Tezgör, Julie Marchand, Christiane Römer-Strehl, La nécropole hellénistique de Plinthine, FIFAO, Le Caire, Ifao, sous presse.

2. Marie-Françoise Boussac, Sylvain Dhennin, Bérangère Redon, Claire Somaglino, Gaëlle Tallet (éd.), Western Borders and Margins of Egypt from Antiquity to the Middle Ages/Frontières et marges occidentales de l'Égypte de l'Antiquité au Moyen Âge, actes du colloque international, Le Caire, 2-3 décembre 2017, Le Caire, Ifao, manuscrit soumis en avril 2020.

\subsection{Articles (sous presse ou soumis en 2020)}

1. Marie-Françoise Boussac, Bérangère Redon, «The Mareotis Area. Integration of a Marginal Territory into Egypt through the Wine Production ", in Katherine Blouin (éd.), Imperial Landscapes: Empires, Societies, and Environments in the Ancient to Modern Nile Delta, à paraittre.

2. Marie-Françoise Boussac, Bérangère Redon, «Définition mouvante et logique d'appropriation des confins nord-ouest de l'Égypte du Nouvel Empire à l'époque romaine. À propos des découvertes archéologiques récentes à Plinthine et Taposiris Magna ", in MarieFrançoise Boussac, Sylvain Dhennin, Bérangère Redon, Claire Somaglino, Gaëlle Tallet (éd.), Western Borders and Margins of Egypt from Antiquity to the Middle Ages/Frontières et marges occidentales de l'Égypte de l'Antiquité au Moyen Âge, actes du colloque international, Le Caire, 2-3 décembre 2017, Le Caire, Ifao, manuscrit soumis en avril 2020.

3. Mikaël Pesenti, «Quelques données sur la place et le rôle de Plinthine/Kom el-Nogous dans les échanges entre Égypte et Méditerranée à l'époque saïto-perse. Étude du mobilier céramique d'un territoire en marge ", in Marie-Françoise Boussac, Sylvain Dhennin, Bérangère Redon, Claire Somaglino, Gaëlle Tallet (éd.), Western Borders and Margins of Egypt from Antiquity to the Middle Ages/Frontières et marges occidentales de l'Égypte de l'Antiquité au Moyen Âge, actes du colloque international, Le Caire, 2-3 décembre 2017, Le Caire, Ifao, manuscrit soumis en avril 2020.

4. Joachim Le Bomin, Julie Marchand, Aude Simony, « Long and Middle Distance Trade in the Occidental Delta During Late Antiquity: The Taposiris Magna and Kom Abu Billu Examples ", in Valentina Caminneci, Maria Concetta Parello, Maria Serena Rizzo (éd.), LRCW6: 6th International Conference on Late Roman Coarse Ware, Cooking War and Amphorae in the Mediterranean: Archaeology and Archaeometry Land and Sea: pottery routes, à paraître.

5. Joachim Le Bomin, Julie Marchand, «Saqia Pots from Taposiris Magna », in in Valentina Caminneci, Maria Concetta Parello, Maria Serena Rizzo (éd.), LRCW 6: 6th International Conference on Late Roman Coarse Ware, Cooking War and Amphorae in the Mediterranean: Archaeology and Archaeometry Land and Sea: pottery routes, à paraître.

6. Julie Marchand, «Il ne faut pas mettre toutes les eulogies dans le même panier! Nouvelles eulogies de Saint Mênas découvertes à Taposiris Magna ", Antiquité Tardive, soumis en juillet 2020.

7. Maël Crépy, Marie-Françoise Boussac, « Western Mareotis Lake(s) during the Late Holocene (4th c. BCE - 8th c. CE): Diachronic Evolution and Evidences for the Digging of a Canal Complex During the Early Roman Period », E\&G Quaternary Science Journal, à paraître. 
8. Maël Crépy, Marie-Françoise Boussac, Joachim Le Bomin, Julie Marchand, Alexandre Rabot, "A Port Out of the Water in the Lower Town of Taposiris Magna (Egypt), Socioenvironmental Trajectories from the 2nd Century BC to the 4th Century AD ", soumis le 16 décembre 2020 à Quaternary International.

9. Marie-Françoise Boussac, Maël Crépy, Thibaud Fournet, Joachim Le Bomin, Julie Marchand, Alexandre Rabot: "Taposiris Magna and its Harbour: New Data on its Chronology and Layout During Antiquity (from Ptolemaic to Early Islamic Period)», soumis pour le livre Egyptian Riverine Harbours, édité par Marine Yoyotte, HARCO Willems and Irene ForstnerMüller.

\subsection{Communications}

1. 25/09/2020 : Mikaël Pesenti, « Material traces of Weaving Activities at Plinthine (Mariout)», visio-conférence Programme de l'Ifao « Mobiliers d'Égypte, Loom \& Weights », organisé par Pascale Ballet et MARIA Mossakowska-Gaubert.

2. 28/09/2020: Bérangère Redon, "Le bâtiment BAT 603 de Plinthine: un entrepôt multiusage?", Journée d'étude "Architecture et techniques de construction des bâtiments et aménagements de stockage en Égypte et au Soudan anciens ", organisée par Adeline Bats et Nadia Licitra, Sorbonne Université.

3. 04/11/2020 : Julie Marchand, «Accueillir les communautés locales : nouvelle réflexion sur les eulogies de saint Mênas d'après les découvertes de Taposiris Magna (Égypte) », Séminaire commun axes A et C, Laboratoire HiSoMA, MOM, Lyon, organisé par Catherine Broc Schmezer et Sylvain Dhennin.

\subsection{Prix, financements et travaux universitaires}

\subsubsection{Prix}

Le Label Archéologie 2020-2021 de l'Académie des inscriptions et belles-lettres a été décerné à la mission française de Taposiris Magna et Plinthine en janvier 2020 : https:// www.aibl.fr/fouilles-archeologiques/labels-archeologie-2020-2021/article/missionarcheologique-francaise-de.

\subsubsection{Financements, projets, mécénats}

- La mission de Taposiris Magna a reçu en 2020 un financement extérieur exceptionnel de la part de la fondation Shelby White and Leon Levy dans le cadre du Shelby White and Leon Levy Program for Archaeological Publications (https://whitelevy.fas.harvard.edu/taposirismagna-egypt-harbor-mareotis-lake).

- Comme en 2018 et 2019, la MFTMP a reçu en 2020 le soutien du fonds Arpamed pour appuyer ses travaux sur la viticulture à Plinthine (https://www.arpamed.fr/projets/projets-2021/ plinthine/).

- B. Redon a participé à la rédaction du projet Nile's Earth, déposé auprès de l'ANR en octobre 2019 (dir. Thierry Joffroy, École d'architecture de Grenoble). Le projet a été classé lors de la première session de sélection, mais n'a finalement pas été retenu pour financement. Un second dépôt a été effectué en novembre 2020.

\subsubsection{Travaux universitaires et contrats}

- M. Crépy a obtenu un renouvellement de son contrat post-doctoral dans le cadre du projet ERC Desert Networks de décembre 2019 à juin 2020. 
- M. Crépy a été classé $2^{\mathrm{e}}$ sur le poste de membre scientifique de l'Ifao, porté au concours en juin 2020.

-T. Faucher a soutenu son HDR intitulée «Une archéologie de la monnaie égyptienne », en décembre 2020, sous la direction de F. de Callataÿ (EPHE). Le mémoire inédit proposé par T. Faucher et intitulé «Circulez! La monétarisation de l'Égypte ptolémaïque et romaine » comprend l'analyse du matériel numismatique mis au jour à Taposiris et Plinthine depuis le début des travaux de la mission, en 1998.

- Paul François a été classé $1^{\mathrm{er}}$ sur le poste d'Ingénieur de Recherche du Laboratoire LA3M. Il a pris son poste au début de l'année 2021. Sa nouvelle affectation ne remet pas en cause sa participation au projet pour les prochaines années.

- Patrice Georges, archéoanthropologue à l'INRAP, responsable de la fouille de la nécropole de Plinthine de 2002 à 2016 a soutenu sa thèse le 26/09/2020, intitulée «La pourriture escamotée : cachez ce cadavre que je ne saurais voir! Quelques destins post mortem de la protohistoire à nos jours à la lumière de l'archéo(thanato)logie : étudier les os, appréhender le corps » (sous la direction de François-Xavier Fauvelle, professeur au Collège de France).

-J. Le Bomin a été classé $3^{\mathrm{e}}$ sur le poste d'Ingénieur de Recherche de l'USR CEAlex.

- Nicolas Morand a obtenu une bourse de la Fondation Fyssen pour mener des travaux postdoctoraux intitulés « Du désert au delta, évolution des pratiques de subsistance des populations humaines dans le delta du Nil et ses marges durant l'Antiquité (vII ${ }^{\mathrm{e}}$ s. av. J.-C. $\mathrm{VII}^{\mathrm{e}}$ s. apr. J.-C.) : une approche archéozoologique». Ils porteront en grande partie sur les restes fauniques de Taposiris et Plinthine.

- J. Marchand a obtenu un contrat de 15 mois dans le cadre du projet H2020 Prevision (septembre 2019 - novembre 2020).

- M. Vanpeene a été classé $1^{\mathrm{er}}$ sur le poste d'Ingénieur de Recherche de l'USR CFEETK. Il a pris son poste au début de l'année 2021. Sa nouvelle affectation ne remet pas en cause sa participation au projet pour les prochaines années.

- M. Vanpeene a soutenu sa thèse (dir. Laurent Coulon, EPHE, PSL, Pierre Zignani, IRAMAT) le 23 janvier 2021.

\section{BIBLIOGRAPHIE}

AMS 1958

Army Map Service, Burg El Arab, NH35-8, 1958.

ARROWSMITH, 1807

Aaron Arrowsmith, Map of Lower Egypt, London, Arrowsmith, 1807.

BLUE, KHALIL 2011

Lucy Blue, Emad Khalil, A Multidisciplinary Approach to Alexandria's Economic Past: The Lake Mareotis Research Project, BAR-IS 2285, Oxford, BAR Publishing, 2011.

BONHOMME et al. 2014

Vincent Bonhomme, Sandrine Picq, Cédric Gaucherel, Julien Claude, « Momocs: Outline Analysis Using R », Journal of Statistical Software 56, 2014, p. 1-24. 
BOUCHAUD, REDON 2017

Charlène Bouchaud, Bérangère Redon, « Heating the Greek and Roman Baths in Egypt.

Papyrological and Archeobotanical Data ", in Bérangère Redon (éd.), Collective Baths in Egypt 2: New Discoveries and Perspectives, EtUrb 10, Le Caire, Ifao, 2017, p. 323-349.

BOUSSAC et al. soumis

Marie-Françoise Boussac, Maël Crépy, Thibaud Fournet, Joachim Le Bomin, Julie Marchand, Alexandre Rabot, « Taposiris Magna and its Harbour: New Data on its Chronology and Layout During Antiquity (from Ptolemaic to Early Islamic Period) », soumis pour Egyptian Riverine Harbours, édité par Marine Yoyotte, Harco Willems and Irene Forstner-Müller, Le Caire, Ifao, à paraître.

CHABROL, LANCRET 1829

Gaspard de Chabrol, Marie-Ange Lancret, « Notice topographique sur la partie de l'Égypte comprise entre Rahmanyeh et Alexandrie, et sur les environs du lac mareotis ", in Description de l'Égypte, t. XVIII/2, Paris, 1829, Panckoucke, p. 3-28.

COSTE 1827

Pascal-Xavier Coste, Carte de la Basse-Égypte dédiée à Mohammed Aly Pacha, vice Roi, Paris, Picket, 1827.

CRÉPY, BOUSSAC 2021

Maël Crépy, Marie-Françoise Boussac, « Western Mareotis Lake(s) During the Late Holocene (4th c. BCE - 8th c. CE): Diachronic Evolution and Evidences for the Digging of a Canal Complex During the Early Roman Period. », E\&G Quaternary Science Journal 70, 2021, p. 39-62.

DE COSSON 1935

Anthony De Cosson, Mareotis: Being a Short Account of the History and Ancient Monuments of the NorthWestern Desert of Egypt and of Lake Mareotis, Londres, Country Life, 1935.

DENON 1803

Vivant Denon, Voyage dans la Basse et Haute Égypte, Paris, Didot, 1803.

AL-FALAKI 1866

Mahmoud-Bey al-Falaki, Carte des environs d'Alexandrie : contenant le lac Maréotis, ceux d'Aboukir et d'Edkou ainsi que les anciens cours d'eaux et les villes dont les emplacements y sont déterminés par mes propres recherches, 1866.

AL-FALAKI 1872

Mahmoud-Bey al-Falaki, Mémoire sur l'antique Alexandrie, ses faubourgs et environs découverts par les fouilles, sondages, nivellements et autres recherches faits d'après les ordres de S.A. Ismail pacha, vice-roi d'Égypte, par Mahmoud-Bey, Copenhague, Imprimerie de B. Luno, 1872.

EMPEREUR, PICON 1998

Jean-Yves Empereur, Maurice Picon, « Les ateliers d'amphores du lac Mariout », in Commerce et artisanat dans l'Alexandrie hellénistique et romaine. Actes du Colloque d'Athènes, BCH-suppl. 33, Athènes, EFA, 1998, p. 75-79.

FLAUX 2011

Clément Flaux, « Connexion de la région lagunaire d'Alexandrie au Nil depuis 2000 ans : entre contrôle anthropique et forçage naturel », Méditerranée 117, 2011, p. 73-79.

FLAUX 2012

Clément Flaux, Paléo-environnements littoraux Holocène du lac Maryut, nord-ouest du delta du Nil, Égypte, thèse de doctorat, Aix-Marseille Université, 2012. 
FLAUX et al. 2017

Clément Flaux, Nick Marriner, Mena el-Assal, David Kaniewski, Christophe Morhange, « Late Holocene Erosion of the Canopic Promontory (Nile Delta, Egypt) », Marine Geology 385, 2017, p. 56-67.

GoDLIEWSKA 1988

Anne Godliewska, The Napoleonic Survey of Egypt: A Masterpiece of Cartographic Compilation and Early Nineteenth-century Fieldwork, Cartographica 25/1-2, Toronto, Winters College York University, 1988.

HAGUET 2007

Lucile Haguet, Aegyptus, l'Égypte de l'Occident. Concept et représentation de l'Égypte dans la cartographie occidentale $d u X^{e}$ au XVIII siècle, thèse de doctorat, université Paris-Sorbonne, 2017. JACOTIN 1818

Pierre Jacotin, Carte topographique de l'Égypte et de plusieurs parties des pays limitrophes, Paris, 1818. JACOTIN 1824

Pierre Jacotin, « Mémoire sur la construction de la carte de l'Égypte », in Description de l'Égypte, t. XVII, Paris, Panckoucke, 1824, p. 437-652.

LAVAINE et al. 2011

Catherine Lavaine, André Evette, Hervé Piégay, Bernard Lachat, Patrice Bahic, « Les Tamaricaceae en génie végétal ", Sciences Eaux \& Territoires. La revue d'Irstea, hors-série 4, 2011, article en ligne, https://doi.org/10.14758/SET-REVUE.2011.HS.04, consulté le 30 mai 2021.

LE PÈRE 1823

G. Le Père, « Nome Maréotique », in Dictionnaire des Découvertes XII, Paris, Louis Colas, 1823, p. 214-222.

LE PÈRE 1825

G. Le Père, «Extrait d'un Mémoire sur les lacs et les déserts de la Basse Égypte », in Description de l'Égypte, t. XVI, Paris, Panckoucke, 1825, p. 199-227.

LE PÈrRE 1829

G. Le Père, « Mémoire sur la partie occidentale de la province de Bahyreh, connue anciennement sous le nom de nome maréotique ", in Description de l'Égypte, t. XVIII/2, Paris, 1829, p. 29-57.

MORHANGE et al. 2015

Christophe Morhange, Nick Marriner, Maria Luisa Blot, Guénaëlle Bony, Nicolas Carayon, Pilar Carmona, Clément Flaux, Matthieu Giaime, Jean-Philippe Goiran, Mourad Kouka, Anna Lena, Ameur Oueslati, Marinella Pasquinucci, Alexeï Porotov, « Dynamiques géomorphologiques et typologie géoarcheologique des ports antiques en contextes lagunaires », Quaternaire 26/2, 2015, p. 117-139.

NENNA et al. 2020

Marie-Dominique Nenna, Aude Simony, Kathrin Machinek, Georges Soukassian, Valérie Pichot, Ismaël Awad, Romain Séguier, Mohamed Abdelaziz, Mohamed Elsayed, Isabelle Hairy, Philippe Soubias, "Alexandrie (actions du Centre d'études alexandrines) », Bulletin archéologique des Écoles françaises à l'étranger, 2020, article en ligne sur OpenEdition Journals, https:// journals.openedition.org/baefe/1094, consulté le 30 mai 2021.

OLIVER 1945

F.W. Oliver, « Dust-Storms in Egypt and Their Relation to the War Period, as Noted in Maryut, 1939-45 », The Geographical Journal 106/1-2, 1945, p. 26. 
RECLUS 1885

Élisée Reclus, L’Afrique Septentrionale: le Bassin du Nil, Nouvelle Géographie Universelle 10, Paris, Hachette, 1885.

RENNELL 1800

James Rennell, The Geographical System of Herodotus, examined, London, Bulmer and Co, 1800.

RODRIGUEZ, MORRIS, BELZ 2005

Ernesto Rodriguez, Charles S. Morris, Eric J. Belz, A Global Assessment of the SRTM Accuracy: The Shuttle Radar Topography Mission, Data Validation and Applications Workshop, Virginia, Reston, 2005.

ST JOHN 1849

Bayle St John, Adventures in the Libyan Desert and the oasis of Jupiter Ammon, New York, London, Putnam, John Murray, 1849.

ST JOHN 1850

Bayle St John, Map to Illustrate a Journey from Alexandria (Egypt) to The Oasis of Siwah: Performed in the Autumn of 1847, Paris, [s.n.], 1850.

SURVEY OF EGYPT 1910

Survey of Egypt, Mudîrîyet El- Beheira, Le Caire, Survey Department Egypt, 1910.

SURVEY OF EGYPT 1942

Survey of Egypt, El Hammam 88/42, Le Caire, Survey Department Egypt, 1942.

TRONCHÈRE et al. 2014

Hervé Tronchère, Bertrand Millet, Jean-Philippe Goiran, Pierre Carbonel, Hatem Djerbi, Ruben Vera, Magdy Torab, Mena Elassal, Yan Callot, « Geoarchaeological Results from the Harbor of Taposiris and Implications Concerning the Construction of the Harbor ", ÄgLev 22-23, 2014, p. 383-394.

TRONCHÈRE 2010

Hervé Tronchère, Approche paléoenvironnementale de deux sites archéologiques dans le delta du Nil: avaris et la branche Pélusiaque, Taposiris et le lac Mariout, thèse de doctorat, université Lumière Lyon 2, 2010.

WARNE, STANLEY 1993

Andrew G. Warne, Danil Jean Stanley, « Late Quaternary Evolution of the Northwest Nile Delta and Adjacent Coast in the Alexandria Region, Egypt », Journal of Coastal Research 9/1, 1993, p. 26-64.

WORONKO 2012

Barbara Woronko, « Late-Holocene Dust Accumulation within the Ancient Town of Marea (Coastal Zone of the South Mediterranean Sea, N Egypt) », Quaternary International 266, 2012, p. 4-13.

\section{NOTES}

1. CRÉPY, BOUSSAC 2021.

2. BoussAC et al. soumis.

3. CRÉPY et al. soumis.

4. DENON 1803, pl. VI, 1. 
5. Par exemple, WARNe, STANLEY 1993 ; TRONCHÈRe et al. 2012; FlAUX 2012 ; WorONKo 2012 ; MORHANGE et al. 2015 ; FlAUX et al. 2017.

6. En particulier issues de BLUE, KHALIL 2011 et des rapports rendant compte de la prospection du programme GEOMAR conduit par le CEAlex, dans NENNA et al. 2020.

7. Tableaux 2-4 et données publiées par EMPEREUR, PICON 1998 ; WARNE, STANLEY 1993 ; TRONCHÈRE 2010 ; BLUE, KHALIL 2011 ; et TRONCHÈRE et al. 2012.

8. Pour plus d'informations, voir Crépy et Boussac (2021) pour l'échelle régionale et Crépy et al. (soumis) pour les conséquences environnementales de l'implantation du port, en particulier la salinisation du milieu

9. BLUE, KHALIL 2011

10. BOUCHAUD, REDON 2017

11. BonHomme et al. 2014.

12. LAVAINE et al. 2011.

13. BOUCHAUD, REDON 2017.

14. RODRIGUEZ, MORRIS, BELZ 2005.

15. https://sketchfab.com/3d-models/fouloir-saite-de-plinthineegypte-9d2c66715fde4d898874be320f5c7a10, consulté le 26 mai 2021.

INDEX

Thèmes : IFAO

nature https://ark.frantiq.fr/ark:/26678/pcrtb1E0Dz7cSX

Année de l'opération : 2020

chronologie https://ark.frantiq.fr/ark:/26678/pcrtbm27waEaeg, https://ark.frantiq.fr/ark:/ 26678/pcrtYHaws8Bjft, https://ark.frantiq.fr/ark:/26678/pcrtM6FrOydySh anthroponymes https://ark.frantiq.fr/ark:/26678/pcrt5XAzjouWxy sujets https://ark.frantiq.fr/ark:/26678/pcrtihlJWfvH4v, https://ark.frantiq.fr/ark:/26678/ pcrtYhYMiLwDUr, https://ark.frantiq.fr/ark:/26678/pcrtWegewfitfX, https://ark.frantiq.fr/ ark:/26678/pcrtVemT606YVG, https://ark.frantiq.fr/ark:/26678/pcrtb1E0Dz7cSX, https:// ark.frantiq.fr/ark:/26678/pcrtPe1qxjEkcR

lieux https://ark.frantiq.fr/ark:/26678/pcrthIZrdSImP3, https://ark.frantiq.fr/ark:/26678/ pcrtsgWZ4lzKyf

\section{AUTEURS}

\section{BÉRANGÈRE REDON}

Historienne, archéologue et directrice de la mission, CNRS, UMR 5189 


\section{MARIE-FRANÇOISE BOUSSAC}

Historienne et ancienne directrice de la mission, université Paris Nanterre

ESSAM AHMED

Archéobotaniste, National Museum of Egyptian civilization - NMEC

CHARLÈNE BOUCHAUD

Archéobotaniste, CNRS, MNHN

\section{MAËL CRÉPY}

Géographe et géomorphologue, CNRS, UMR 5189

SYLVAIN DHENNIN

Archéologue et égyptologue, CNRS, UMR 5189

MENNAT-ALLAH EL DORRY

Archéobotaniste, MoTA

\section{PAUL FRANÇOIS}

Architecte et ingénieur, université de Nantes

\section{LOUIS DAUTAIS}

Archologue, université Paul-Valéry Montpellier 3, université catholique de Louvain

\section{JOACHIM LE BOMIN}

Archéologue, université Paris 1 Panthéon-Sorbonne

\section{LOUIS MANIÈRE}

Ingénieur au sein du projet Desert Networks (CNRS), spécialiste de SIG

JULIE MARCHAND

Céramologue, CNRS, UMR 5189

\section{SÉVERINE MARCHI}

Archéologue, CNRS, UMR 8167

\section{CLÉMENCE PAGNOUX}

Archéobotaniste, École française d'Athènes

MIKAËL PESENTI

Céramologue, Aix-Marseille Université

\section{ALEXANDRE RABOT}

Spécialiste de SIG, UMR 5189/université Lumière Lyon 2

GONCA ŞENOL

Amphorologue, université d'Izmir 


\section{CLAIRE SOMAGLINO}

Archéologue et égyptologue, université Paris Sorbonne, UMR 8167

MATTHIEU VANPEENE

Architecte, Ifao/EPHE

NOÉMI VILLARS

Data manager 